\title{
Review Article \\ The Extraordinary Progress in Very Early Cancer Diagnosis and Personalized Therapy: The Role of Oncomarkers and Nanotechnology
}

\author{
Marialuigina Fruscella, ${ }^{1,2}$ Antonio Ponzetto, ${ }^{3}$ Annalisa Crema, ${ }^{1}$ and Guido Carloni ${ }^{1}$ \\ ${ }^{1}$ Institute of Translational Pharmacology, National Research Council (CNR), Rome, Italy \\ ${ }^{2}$ Department of Human and Social Science, Headquarters, National Research Council (CNR), Rome, Italy \\ ${ }^{3}$ Department of Medical Sciences, University of Torino, Torino, Italy \\ Correspondence should be addressed to Guido Carloni; guido.carloni@ift.cnr.it
}

Received 18 December 2015; Accepted 21 February 2016

Academic Editor: Aws Alshamsan

Copyright (C) 2016 Marialuigina Fruscella et al. This is an open access article distributed under the Creative Commons Attribution License, which permits unrestricted use, distribution, and reproduction in any medium, provided the original work is properly cited.

The impact of nanotechnology on oncology is revolutionizing cancer diagnosis and therapy and largely improving prognosis. This is mainly due to clinical translation of the most recent findings in cancer research, that is, the application of bio- and nanotechnologies. Cancer genomics and early diagnostics are increasingly playing a key role in developing more precise targeted therapies for most human tumors. In the last decade, accumulation of basic knowledge has resulted in a tremendous breakthrough in this field. Nanooncology, through the discovery of new genetic and epigenetic biomarkers, has facilitated the development of more sensitive biosensors for early cancer detection and cutting-edge multifunctionalized nanoparticles for tumor imaging and targeting. In the near future, nanooncology is expected to enable a very early tumor diagnosis, combined with personalized therapeutic approaches.

\section{Introduction}

Personalized oncology provides new hopes for reducing cancer mortality by selectively targeting anticancer drugs to malignant cells, at the level of the target-sites on the cell surface and within the tumor microenvironment $[1,2]$. Nanooncology has succeeded in increasing specificity and effectiveness of cancer treatments, both facilitating drugs uptake and delivery and reducing systemic toxicity and adverse events.

Molecular diagnostics based on the development in sequencing and genotyping of tumor DNA plays a crucial role in personalized therapy [3]. Mutations are a prerequisite for tumor development, often found in cell signaling proteins as a result of deletions/point mutations in gene-coding regions or in promoter-enhancer sequences, DNA insertions, copy-number variations, and chromosomal translocations. However, not every mutation leads to cancer. Somatic mutations are typically present in the tyrosine kinase (TK) domains within DNA sequences encoding for growth factor receptors involved in signaling pathways, that is, the EGFR, HER2/ERBB2, KRAS, KIT, FGFR, VEGF-R, ABL, PI3K, mTOR, PDGF-R, and HGFR/MET. The activating mutations of the EGFR in a subset of patients with advanced non-smallcell lung cancer (NSCLC) who respond to the EGFR tyrosine kinase inhibitor (TKI) gefitinib or erlotinib are paradigmatic [4].

Personalized oncology started with the HER2 antibody trastuzumab in breast cancer, followed by the BCR-ABL1 inhibitor imatinib in chronic myeloid leukemia (CML) and EGFR-TKIs and ALK-MET inhibitor crizotinib in NSCLC; the most recent success is the BRAF inhibitor vemurafenib in melanoma [1].

The dramatic breakthroughs in the clinical use of TKIs for cancer treatment depended on the predictive specific biomarkers. Metastatic colorectal cancers (CRC), expressing EGFR, allow for predictive response to targeted therapy with specific anti-EGFR monoclonal antibodies (mAbs) [5, 6]; however, CRC carrying KRAS mutations failed to respond. 
In addition, current cancer immunotherapy is predominantly based on antitumor specific mAbs [5], after failure of interleukin-2 (IL-2), and the potentially lifethreatening adverse events of adoptive cell-transfer of autologous tumor-infiltrating reactive lymphocytes; despite this, therapy achieved $40 \%$ tumor regression in advanced metastatic melanoma [7].

The checkpoint regulators CTLA- 4 and PD-1 expressed in immune cells were successfully targeted for immunotherapies: in metastatic melanoma patients the anti-CTLA-4 ipilimumab significantly extended the median OS to $10.1 \mathrm{~m}$, in phase III, compared to $6.4 \mathrm{~m}$ for the control group, enabling an unprecedented durable tumor regression of $46,6 \%$ at $1 \mathrm{y}$. and $23,5 \%$ at $2 \mathrm{y}$. [8]. Ipilimumab is becoming a firstline treatment, doubling survival at $1-2 \mathrm{y}$. in the metastatic melanoma; its association with anti-PD-1 mAb nivolumab ameliorated the 2-year survival to $80 \%$, in phase I [9].

Nanotechnology is revolutionizing cancer management, because it can partially change its diagnosis, clinical course, and prognosis. The cancer molecular profiling before treatment can be highly prognostic and predictive of therapeutic responses or recurrence, by allowing the most suitable treatment for each individual cancer to be administrated.

\section{Molecular Diagnostics for Personalized Oncology}

Mutations in TK genomic domains of a number of growth factor receptors were dubbed "driver" mutations, as they induce malignant transformation. Despite initial successes, targeted therapy may fail in a proportion of patients, especially in tumors with driver mutations. Genetic heterogeneity and insurgence of drug resistance due to secondary mutations, and tumor relapse, may occur, either early or late during tumor development, in a minority of tumor cell subclones, which engender insensitivity to the drug and tumor progression.

Examples are BRAF and MEK mutations in melanoma [10] or gatekeeper mutations of EGFR, such as pT790M mutation in NSCLC, that causes reduced drug binding and may coexist with EGFR pT790M and MET amplification, and the P13KCA mutation or overexpression of GAS6 ligand and its homologous receptor AXL [1]. The resistance to EGFR TK inhibitors in EGFR-mutated NSCLC patients not correlated to PT790M mutation is modulated by FAS and $\mathrm{NF} \kappa \mathrm{B}$ signaling [11].

The understanding of metabolic and signaling pathways implicated in most of the encountered resistance mechanisms is becoming clear, hence allowing development of new therapeutic approaches.

Advanced melanoma, which is particularly resistant to chemotherapy and rapidly metastasizes, is representative of a multidrug resistant (MDR) cancer type. BRAF-V600E mutations have been found in over $60 \%$ of cases of melanoma. Although initially responsive to specific inhibitors, resistance rapidly develops in the form of mutated gene amplification. BRAF is a component of MAPK pathway, which relays proliferative signals, triggered at cell membrane receptors, through the cytoplasm to the nucleus.
The mitogen-activated protein kinase MEK is also commonly overactivated in melanoma; thus, a combination of BRAF and MEK inhibitors was conceived as dual mechanisms to inhibit the MAPK pathway in BRAF-V600 mutation-bearing melanomas. Monotherapy with vemurafenib or dabrafenib achieved OR of $57 \%$ and $50 \%$ and progressionfree survival (PFS) of $6.9 \mathrm{~m}$ and of $5.1 \mathrm{~m}$, respectively, in phase III clinical trials. Dual-therapy consisting of dabrafenib + trametinib obtained a good OR of $75 \%$ and PFS of $9.4 \mathrm{~m}$ in phase II $[12,13]$. Nevertheless, failure to respond and dual resistance to both drugs rapidly developed $[14,15]$.

Prognostic markers assays are relevant in molecular diagnostics for many types of leukemia and cancer (Table 1). Among these, there are the antioncogene TP53, the IGHV mutations, and the expression of CLLUI in chronic lymphocytic leukemia (CLL) and in breast cancer, to predict the recurrence risk of lymph node-negative tamoxifen-treated patients, or methods to quantify estrogen levels and expression of progesterone receptors, HER2 and Ki-67, which also have prognostic value [1]. Cell surface receptors (EGFR, HER2, and KIT) are useful targets for therapy, whereas the cytoplasmic BRAF results are less accessible.

Mutations of surface receptors are often present in NSCLC. EGFR mutations are in-frame with microdeletions at exon 19 [16] and rearrangement of the EML4 gene with parts of the ALK gene results in enhanced proliferation and transforming activity in vitro [17]. KRAS mutations are usually in codon 1 or 12 , whereas the vast majority of BRAF mutations correspond to a hotspot transversion mutation T1799A at exon 15. All the above-mentioned mutations are key predictive biomarkers for developing effective strategies of targeted therapy.

The role of noncoding DNA in cancer has revealed novel types of promising biomarkers. Genetic and epigenetic biomarkers in cancer management are beginning to affect prognosis and outcome for most human tumors [18].

2.1. Genetic Oncomarkers in Tumor and Metastasis Detected by Advanced Genomics. Therapeutic targeting in solid tumors is primarily based on the identification of cancer-specific gene mutations [19]. Targeted delivery of drugs specifically directed to individual tumor cells resulted in successful treatment of NSCLC displaying EGFR mutations or carrying the EGFR-amplified gene. NSCLC patients treated with gefitinib showed a significant survival increase [20] whereas patients with small-cell lung carcinoma (SCLC) failed to respond. Integration of new proteomics assays for identifying biomarkers with statistical analysis for standardizing clinical data resulted in the implementation of personalized medicine, both providing a predictive response to treatments and indicating the likelihood of adverse events [21].

NSCLC frequently express genomic mutations in HER2 (28\%), BRAF (2-5\%), ALK (5\%), and KRAS (30\%) [22-25]. The response to specific TKIs varies according to tumor type and genetic makeup. Tyrosine kinases are undergoing evaluation as first-line treatment alone or combined with chemotherapy and radiotherapy. In NSCLC, FGRG1 amplifications, PK3CA and DDR2 mutations, and ROS1 and 


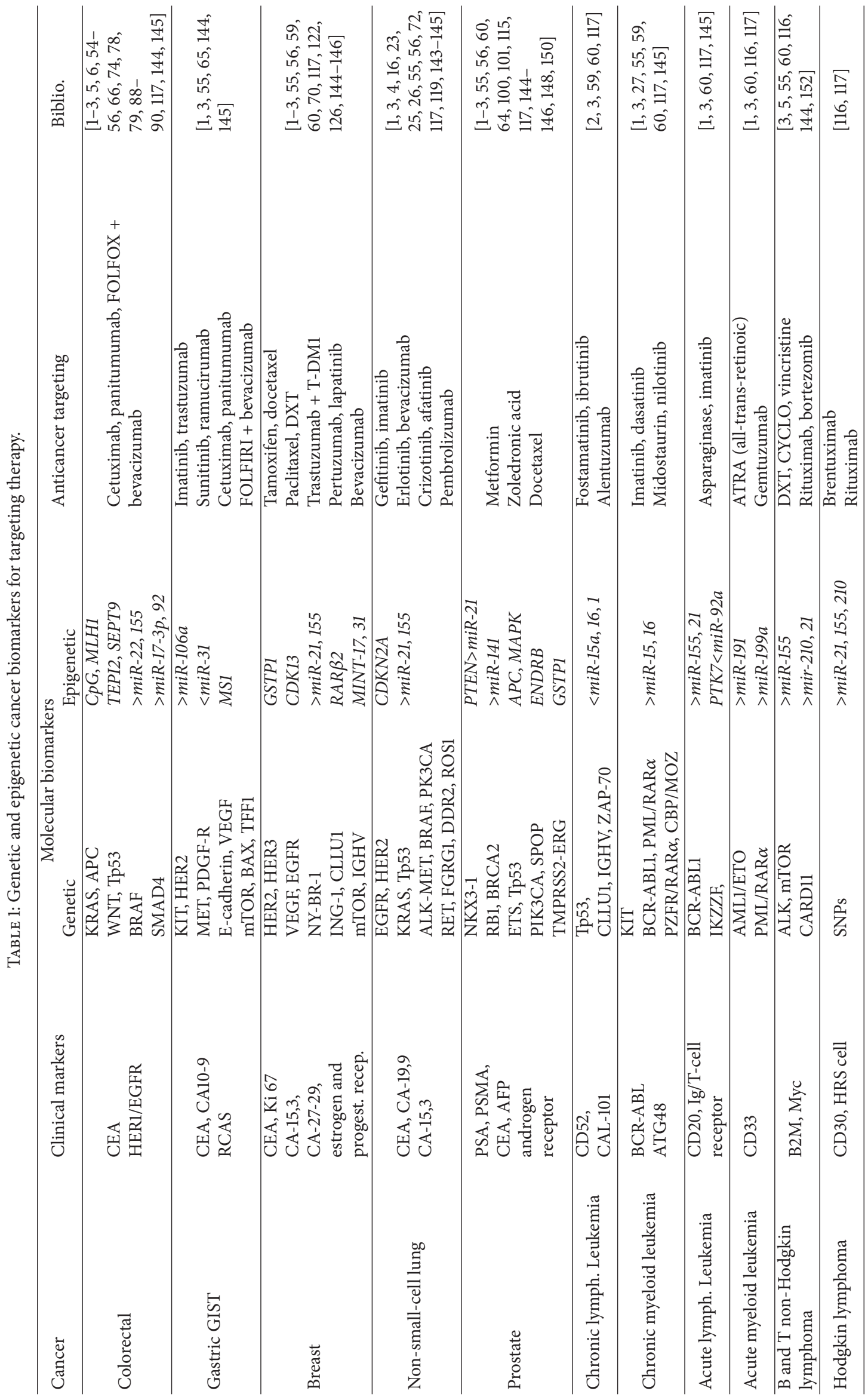




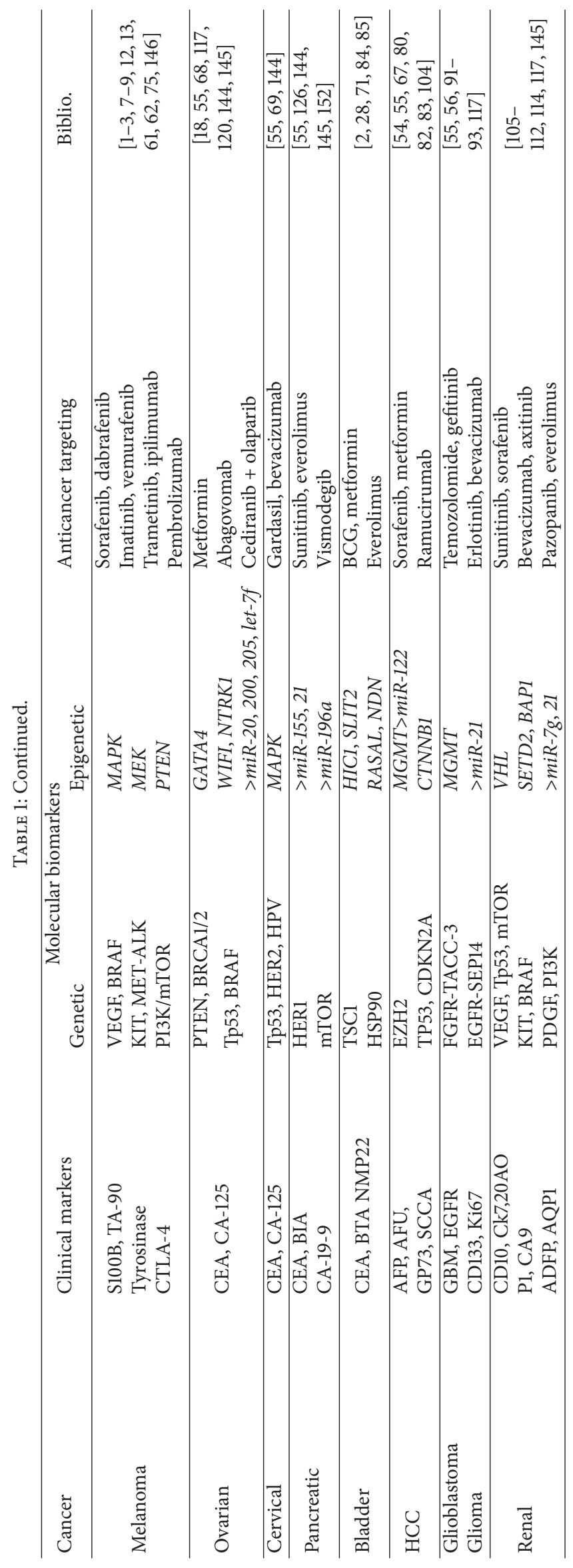


RET rearrangements [26] increase the number of potentially treatable patients.

Targeted therapy improved survival in interstitial stromal gastric cancer presenting KIT mutations and in HER2-overexpressing breast cancer $[1,2]$. In myeloid leukemia, specific KIT-driven mutations in codon 822 are predictive of a positive response to imatinib, while other mutations require different treatments [27]. Everolimus therapy resulted in longer remission in the $5 \%$ of patients with advanced bladder cancer carrying a TSC1 gene mutation [28].

Molecular diagnostics now employs next-generation DNA sequencing [29], that is, oligonucleotide arrays, to reveal transcription profiling and large-scale single-nucleotide polymorphism assays to detect DNA deletions or amplifications [3]. The latest assays for sequencing the complete genome and nanopores [30] are specifically adapted to the clinical requirements, characteristic of a particular cancer. DNAs or RNAs, cDNAs, miRNAs, and small RNAs are specifically selected through novel techniques for genome resequencing and genetic variation. Sequencing of the complete sets of exons of the entire exome in a genome by target-capture hybridization identifies common and rare variants, along with infrequent mutations that are predisposing to cancer. Genome-wide-association studies (GWAS) have enabled investigation of sequence variations, which occur in chromosomal regions from different individuals, and the detection by chip-based methods of single-nucleotide polymorphisms in the human genome [2].

This has facilitated innovative approaches concerning the genesis of cancer and allowed the discovery of BRCA1-2 gene mutations, known to predispose to cancer [3]. Stromal cells are involved in the genesis and regulation of metastasis [31]. Translation of recent discoveries in metastatic cells and their interactions with the stromal cells is required to bypass the main obstacles in cancer therapy [32].

The microenvironment plays a role in renormalization of tumor cells, suggesting that the recovery of normal functions of stromal cells may be an advantageous strategy in cancer treatment [33]. Tumor suppression achieved by the microenvironment of embryonic stem cells has highlighted new potential therapeutic targets [34].

Expression profiling of genes involved in cell-cell and cellmatrix contacts has identified changes involved in metastasis. WNT, Notch, and BMP pathways regulate the dormancy of metastasis [35] and GATA3 inhibits metastasis via miRNA-29b expression [36]. In preclinical models, miRNA126 promotes breast cancer metastasis affecting the tumor microenvironment [37]. Investigating the epithelial mesenchymal transition (EMT), and gene expression changes during transition [38], has enabled the understanding of the modulation of genes involved in ECM remodeling, cell adhesion, inflammation, oxidative stress, BMP, and TGF- $\beta$ and metastasis. This led to the exploration of other adverse events that facilitate malignancy, invasion, and migration, characteristic of tumors with cancer stem-cell- (CSC-) like phenotype, which promotes EMT [39].

EMT promotes resistance to chemo- and radiotherapy; hence, its elucidation has opened the way to therapeutic manipulation of the most aggressive cancers. A robust
EMT-signature was developed to predict resistance to EGFR and P13K/AKT pathway inhibitors in NSCLC patients [40] in whom erlotinib-resistance was dependent on AXL-kinase activation [41]. AXL-inhibitor SGI-7079 reverses erlotinibresistance.

2.2. Targets in Cancer Stem Cells. CSC are a stable cell population, whose origin is still a source of fierce debate, and their golden standards are self-renewal in vitro and in vivo, preserving a constant reservoir. CSC both interact with the tumor microenvironment and ensure to themselves changes that are the ideal niche for their preservation and continuity overtime. Although present in small number in the tumors, CSC show even within the same tumor a heterogeneity that depends on the stage, the degree, and the level of differentiation. CSC act as tumor initiating cells (TIC) and are involved in metastasis and in mediating tumor progression and relapse. A number of markers have been defined that enabled the isolation and characterization of CSC exhibiting different phenotypes in diverse types of tumors $[42,43]$ allowing targeting therapies [44]. CSC are highly carcinogenic and very resistant to conventional anticancer chemotherapy and radiotherapy [45]; hence, specific targets are mandatory for directing anticancer agents for effective therapies to successfully eradicate cancers. CSC are mainly involved in resistance: to DNA damage induced by high levels of DNA repair [46], to drug penetration, by enhanced expression of ATP-binding cassette drug transporters [47], and to apoptosis [48].

The location of CSC in "niches" of the microenvironment and hypoxia protects them from anticancer agents [49]. Various signaling pathways of normal stem cells are also required for the maintenance of CSC renewal in different human malignancies, such as Hyppo, Hedgehog, Notch, WNT, and P13K/AKT [45]. Snail transcription factor and TGF- $\beta$, involved in EMT, play a primary role in CSC activation.

Vitamin D3 and phytochemicals act on the CSC signaling pathway, such as green tea catechins and the salinomycin, which selectively kills breast CSC [50], and provide potentially effective compounds that reduce tumor growth [51]. Chemotherapy that preferentially kills most of the sensitive tumor cells selects the CSC-subset [52]. New agents specifically targeting for CSC are actively sought after [48], but not yet available.

2.3. Searching for Epigenetic Cancer Biomarkers. The longterm disruption of epigenetic control in cancer cells is due to reduced levels of DNA methylation [53], alteration in histones or their acetylation [18], and changes in lncRNAs and miRNAs $[54,55]$. As DNA methylation is the initial control point of regulating gene transcription, as well as controlling antioncogene expression and transcription of silencing RNAs that predispose to cancer, epigenetics is principally implicated in the induction and maintenance of carcinogenesis [56]. The dependency on epigenetic alterations, such as changes in DNA methylation, for the survival of cancer cells, is well known and demonstrated in vivo in preclinical cancer models [18]. In the majority of cancers, loss of DNA methylation is 
prevalent; conversely $\mathrm{CpG}$ islands of tumor suppressing genes (TSGs) are hypermethylated, leading to their silencing [57].

Silencing of regulatory genes has been suggested as alternative to mutations that impair genes structures or inactivate their functionality. Silencing of BRCA1-2 in ovarian and breast carcinomas, and of CDKN2A in squamous cell lung cancer, is typical and supports the "two-hit" hypothesis of Knudson that requires a mutation in both TSGs alleles for their silencing to produce a tumorigenic phenotype [58].

The first evidence for the involvement of miRNA in cancer was revealed by the discovery of frequent deletions of miRNA-15 and miRNA-16 in chronic lymphocytic leukemia (CLL) [59].

Epigenetic personalized medicine has advanced cancer therapy [60]. Epigenetic alterations are increasingly being discovered. Such alterations affect chromatin and gene expression, via DNA methylation, histone acetylation, and deacetylation (e.g., HDAC), respectively, silencing and reactivating gene expression. Indeed, HDAC and DNA methyltransferase (DMNT) inhibitors are successfully employed in MDS and various forms of leukemia [60]. Epigenetic biomarkers have greatly enhanced cancer detection and diagnosis (Table 1), such as for skin [61,62], oral squamous cell carcinoma (OSCC) [63], prostate [64], gastric [65], colorectal [66], liver [67], ovarian [68], cervical [69], breast [70], bladder [71], respiratory tract [72], and lung [23] cancers.

In addition to TSGs, DNA methylation may also silence genes implicated in cell growth, regulation, and differentiation: hundreds of methylated genes have been reported in human cancers, associated with poor disease outcome and clinical response [73]. New methods for DNA methylation fingerprinting have enabled growth of epigenetic marks; nonetheless, very few methylated genes are utilized as cancer biomarkers in diagnostics [74]. Epigenetic modifications in BRAF and RAS genes are frequently observed in deregulation of the MAPK signaling pathway that drives melanoma and can therefore be utilized as genetic cancer biomarkers [75].

Abnormal DNA methylation, histone modifications, and impaired expression of induced miRNAs drive OSCC [76] presenting MLH1 mutations and hypermethylation and silencing of MGMT and RASSF genes. Hypermethylated HS3ST2, NPY, and HOXA9 genes allow early detection of OSCC, while epigenetic deregulation of NOTCH4 identifies disease recurrence [63].

Genetic and epigenetic changes that occur at different steps in normal cells [18] cooperatively determine the "hallmarks of cancer" leading to malignant transformation [77]. The extraordinary variability of prostate cancer observed by large-scale genomic studies is exemplary of molecular features required for stratifying patients into subgroups for predicting the outcome and selecting the most appropriate therapy. Gene expression profiles and related functional therapeutic information define the best therapy [64].

Prostate cancer frequently presents mutations, such as deletions in TSGs, NKX3-1, PTEN, BRCA2, and RB1, and structural genomic rearrangements which may lead to recurrent fusion of the androgen-responsive genes for TMPRSS2 and transcription factors (TFs). This tumor subtype is androgen-independent, displays a more aggressive phenotype, and has an unfavorable prognosis [3]. Several point mutations have been identified in high-risk prostate cancer patients, providing information on the mechanisms that promote genetic instability and on the routes through which specific genes and associated pathways may function as potential targetable biomarkers for new therapies.

CRC is a paradigmatic example of tumor progression toward full malignancy, in which both genetic and epigenetic markers have been identified [78]. Comparative sequence analysis of adenomas, the most frequent precancerous lesion for adenocarcinomas, suggests that mutations arise primarily affecting genes and pathways that control cellular growth and differentiation and transform normal cells into carcinomas capable of metastasis [79]. The WNT pathway is involved in the adenoma-carcinoma-transition, and an inverse relationship has been observed between WNT and the APC gene, which is involved in early transformation to adenomas and is mutated in about $80 \%$ of CRC. APC mutations result in constitutive activation of WNT signaling. In CRC, other mutated oncogenes include KRAS and BRAF, which increase cell proliferation to the same extent as APC mutations, but mutations also occur in SMAD4 and TGFBR2 genes or as $18 \mathrm{q}$ locus deletions. Loss of TGF- $\beta$ signaling transforms the intermediate differentiated adenoma into a full adenoma and drives late events in tumor development, including mutations in TP53, present in 50\% of CRC. Most CRC occur sporadically; however, they may also arise due to genetically transmitted mutations or following inflammatory bowel disease. Subtypes are defined according to molecular features based on the tumor epigenetic traits, namely, microsatellite instability (MSI), chromosomal instability (CIN), MLH1 promoter methylation, and $\mathrm{CpG}$ dinucleotide island methylator phenotype (CIMP) [18]. All three subtypes carry mutations in protein-coding genes in addition to impaired gene functions, and changes in miRNA expression affecting gene expression.

In HCC significant differential methylation has been revealed by genome-wide methylation profiling and pyrosequencing analysis [80]. Large-scale screening of differentially methylated regions in promoter $\mathrm{CpG}$ islands has shown that the changes in methylation particularly affect the signaling networks of BMP4, CDKN2A, GSTP1, and NFATC1, which are all involved in cell development, expression, and death.

A genetic basis for epigenetic disruption in cancer has been universally recognized. It is thus reasonable to assume that disruption of epigenetic control by mutations of key regulators of the extensive transcriptome amplifies the effect of a single genetic alteration. Large-scale sequencing of HCC has revealed mutations in epigenetic regulators, both associated and unassociated with HBV and HCV infections [81]. Mutated genes, most leading to loss of gene function, have been found in nearly all HCC cases [82]. Point mutations and microdeletions were infrequent in the control genes TP53, CDKN2A, and AXIN1, with the exception of CTNNB1, encoding $\beta$-catenin. Epigenetic alterations in TP53 and CTNNB1 genes offer new therapeutic targets and biomarkers; for example, for the deregulated expression of the regulatory gene EZH2 involved in epigenetic silencing of the $\mathrm{WNT} / \beta$ catenin pathway and of TSG miRNA, the former is associated with progression and aggressive phenotype and the latter with 
prometastatic evolution of HCC. Expression of specific miRNAs is also associated with HCV-related HCC-pathogenesis [83], the molecular network of which includes TSGs, namely, TP53, PTEN, and all-trans-RA, whose disruption is directly involved in tumor development.

Cervical carcinoma is another type of cancer associated with viral infection, in which recurrent somatic mutations of the MAPK1 gene are particularly relevant and may be indicative of ERBB2 activation suggesting that selected cervical carcinoma patients could therapeutically benefit from ERBB2 inhibition [69]. Gene expression levels in HPV integration sites are significantly higher in tumors with HPV integration.

Both hypermethylation and mutation of the TSG necdin (NDN) were detected in most primary urothelial carcinomas, accompanying loss of NDN expression [84]. A total of 50\% of transitional bladder cancers show genetic aberration of chromatin-remodeling genes, among which UTX is preferentially altered in low-stages and low-grades cancers, providing implications for classification and diagnostics [85]. In lung adenocarcinomas, chromatin patterns change according to development and progression of the disease [86]; therefore, defining chromatin patterns according to the lung adenocarcinoma lifecycle would provide new reliable epigenetic biomarkers in diagnosis, prognosis of tumor progression, and therapy.

2.4. Novel Emerging Cancer Biomarkers. Protooncogenes play a very important role in growth and development of normal cells; they can, however, mutate and undergo abnormal activation, which progress towards carcinogenesis. This observation led to the discovery of driving mutations in a number of tumors. Among the mutations in several known oncogenes [87] and antioncogenes acting as TSGs, such as TP53 [88], those located in the PK domains of EGFR, HER2, KIT, FLT3, DMNT (3A and 1), and KRAS GTPase are particularly relevant [60].

In leukemia, chromosomal translocations leading to fusion genes with altered gene function are typical (Table 1). The fusion gene BCR/ABL is formed of the BCR region of chromosome 22 with c-ABL of chromosome 9; it occurs in nearly all CML patients and in $20 \%$ of patients with ALL and constitutively activates ABL kinase. Imatinib, which specifically blocks BCR-ABL enzyme inhibiting ALB kinase activation, is one of the greatest successes achieved in CML therapy. In Burkitt's lymphoma, specific translocation of the c-Myc gene (8:14) occurs, leading to increased expression of target genes and inhibition of $\mathrm{NF} \kappa \mathrm{B}$ pathway genes [3]; in another non-Hodgkin lymphoma, mutations affect $\mathrm{NF} \kappa \mathrm{B}$ via CARD11 gene expression. Therefore, drugs specifically targeting $\mathrm{NF} \kappa \mathrm{B}$ have been developed, such as bortezomib (a proteasome inhibitor) considered the first effective treatment against relapsed and refractory lymphoma, along with chemotherapy.

Transforming genetic fusions have also been recently reported for several solid malignancies and colon adenocarcinomas $[89,90]$ representing promising targets for clinically untreatable cancers.

A subset of glioblastomas, containing the transforming fusion gene involving the TK domain of FGFR and the transforming acid coiled-coil domain of TACC [91], can be treated with FGFR kinase inhibitors which corrects the aneuploidy; in mice harboring intracranial FGFR-TACC3 , oral administration of an FGFR TKI inhibits glioma development [92]. Translocations fusing the EGFR codingregion to several partners have been frequently found in glioblastoma as functional fusion gene [93]. EGFR-SEP14 fusions activate STAT3 signaling and confer sensitivity to EGFR inhibitors and independence to mitogens.

Genome-wide sequencing has identified four subgroups within medulloblastoma (the major cause of death from childhood cancer), characterized by unique targetable somatic copy-number aberrations (SCNAs) involving tandem duplication of the SNCAIP gene, recurrent translocations occurring through chromothripsis-like process and leading to PVT1-MYC and PVT1-NDRG1 fusions, and recurrent targeting of TGF- $\beta$ and NF $\kappa$ B signaling [94].

The prognostic role of the MYCN oncogene amplification in neuroblastoma cells has been evaluated in numerous clinical trials [95]. In about $20 \%$ of more than 1000 investigated neuroblastomas, MYCN has been found abnormally expressed. Since both the status and function of the MYCN gene and chromosomal aberrations have been reported to improve risk assessment of patients, it can help selecting the most appropriate therapy for each subgroup of patients. Using high density SNP analysis novel drivers of neuroblastoma development have been revealed, namely, the BARD1 gene variants, which are a high-risk indicator in carriers [96].

With the advent of GWAS and next-generation sequencing, allowing the in-depth study of the neuroblastoma genome, genes associated with tumor progression have been identified: ALK gene, candidate for therapy with smallmolecule inhibitors; mTOR Aurora kinase and TRKB [95]. The LMO1 locus showed aberrant expression in more than $10 \%$ of the most aggressive cases of neuroblastoma, suggesting that this locus polymorphism is associated with susceptibility to tumor development and influences malignant progression [97]. Chromosomal deletions and sequence alterations of the chromatin-remodeling genes ARID1A-B were found in $10 \%$ of neuroblastomas, providing new biomarkers to identify rearranged DNA fragments in sera allowing for minimal residual disease detection and therapeutic follow-up [98]. In addition, methylation of PCDH8 and SFN can be used as outcome indicators in high-risk patients [99]. In $40 \%$ of gliomas, MGMT, involved in DNA repair, was hypermethylated in the promoter region [53] and associated with glioma sensitivity to radiotherapy and alkylating agents. MGMT protects cells against mutations by removing alkyl groups from guanine. These are imported with the alkylating agents nitrosamide 5-hydroxymethylcytosine $(5 \mathrm{HmC})$, catalyzed by three genes (TET1-TET3), with $\alpha$-ketoglutarate as cofactor, under the control of isocitrate dehydrogenases IDH1 and IDH2.

Silencing of TET1 by miRNA leads to a decrease in $5 \mathrm{HmC}$, while IDH1 and IDH2 mutations have been found in glioma, myeloid leukemia, and melanoma and may therefore serve as diagnostic markers in a number of cancers $[56,60]$.

DNA methylation biomarkers are used for early cancer detection but can also predict and/or monitor therapeutic responses and disease recurrence. Examples include 
the tissue-factor pathway inhibitor 2 (TEPI2), useful for screening CRC in stools and hypermethylation of septin 9 (SEPT9) in blood samples [79]. In prostate cancer tissue and urine, DNA methylation biomarkers such as APC or ENDRB, together with serum prostate specific antigen (PSA) and quantitative evaluation of glutathione-S-transferase $\mathrm{p} 1$ (GSTP1), have improved diagnosis [100, 101].

DNA methylation can be also used as a molecular prognostic biomarker in stage I NSCLC. Methylation of p16 CDKNZA and $\mathrm{H}$-cadherin (CDK13), RASFIA, and APC was shown to strongly correlate with early local and mediastinum lymph-nodal recurrences and short survival [72].

2.5. Toward a Very Early Cancer Diagnosis. Very early detection of cancer and timely monitoring of recurrence are of paramount importance in establishing prognosis and outcome of the disease and, in general, determine the cures that patients should undergo. Initially, noninvasive diagnostic tests are carried out, typically the fecal occult blood test (FOBT) for CRC [78], of blood in urine for bladder or renal cancer, or hemoptysis for lung cancer. However, all these symptoms lack specificity, as they are also present in other pathological conditions. Any positive result requires more invasive clinical investigations, undesirable for patients, often posing risks and requiring radiation exposure.

The detection of DNA methylation biomarkers based on nucleic acids analysis allows for early diagnosis, prognosis, and prediction of therapeutic response [56]. The discovery of marker genes of DNA methylation and the understanding of their role in cancer is leading to the development of new, more sensitive, and specific genomic-based assays. The identification of tumor specific DNA methylation at distal sites from the original neoplasia, such as blood and other bodily fluids, is particularly suitable for early diagnosis and clinical management of cancer patients, as it does not require invasive procedures for sample collection. Two CRC-specific methylation tests are now commercially available: the plasma methylated DNA for SEPT9 promoter (ColoVantage) and the fecal methylated DNA for the methylated vimentin gene (Colo Sure). DNA methylation markers offer a powerful diagnostic tool for screening individuals at risk of developing lung cancer [72].

Circulating DNA, either free or complexed to red blood cells, platelets, or nucleosomes, has been found in the blood during the course of various disease states [60] and can be a very early cancer biomarker, whereas its decrease and disappearance could be used to monitor the success of the therapy. One limit comes from that DNA mutations, epigenetic markers, and signaling pathways require sufficient material from the affected tissues.

Although promising as noninvasive cancer biomarkers, analyzing cell-free circulating nucleic acids has not reached clinical routine. A major hurdle remains which is the detection sensitivity, even though amplified genomic DNA and RNA from single cells can be used to characterize rare circulating cancer cells [102].

Circulating tumor cells (CTC) have been shown to be responsible for tumor metastasis and resistance to anticancer therapies. They are indeed highly pathognomonic of the stage of tumor spread and indicative of metastasis in cancer patients. New clinical tests using minute quantities of CTC are ongoing, for early detection at the preneoplastic and/or premetastatic stage, as for prostate [64] and lung cancers [103].

Other molecules, such as lipids, carbohydrates, proteins, and smaller metabolite products related to cancer, can actually be oncomarkers with prognostic value of cancer progression, therapeutic response, and disease outcome [60]. As an example, comparing the emerging biomarkers from exhaled breath, known as volatile organic compounds (VOC), and those of exhaled breath condensate (containing small molecules and nucleic acids) is noninvasive test, allowing a highly specific diagnosis.

Serum miRNAs play a very important role in activation or suppression of target gene expression (Table 1). Its detection is minimally invasive; however, it may not be specific for the indicated cancer $[54,55]$.

AFP is among the known genetic biomarkers for HCC [104] (Table 1); in addition, other four potential protein biomarkers have been identified: ALNL, FLNB, C4A, and AFT. The first two characterize the HCC group before treatment better than AFP and provide available screening biomarkers in other cancers as well [67].

CA9 has been the first reliable diagnostic biomarker of clear cell renal cell carcinoma (ccRCC) [105]; now, for early detection of kidney cancer, the urine biomarkers aquaporin 1 (AQP1) and adipophilin (ADRP) [106] and increased plasma levels of LCP1, NNMT, and NM23A have been evaluated [107]. Kidney cancer biomarkers play an important role in determining diagnosis and prognosis and are predictive for treatment effectiveness and outcome [108], enabling discrimination between the ccRCC and papillary (pRCC) type 1 (with favorable outcome) and type 2 that rapidly metastasizes [109]. Mucin 1, E-cadherin, VEGF-R2 and VEGF-R3, and cytokeratins (CK) 20 and 7 are differentially expressed in type 1 and type 2 pRCCs, and alpha-methylacyl-CoA racemase mRNA is overexpressed in most pRCCs; all are useful diagnostic biomarkers. CD44, Ki67, CA9, and PCNA, which play a role in apoptosis, cell cycle, cell migration, and angiogenesis, offer prognostic value for ccRCCs. Most ccRCCs bear mutations in the VHL gene [110] that lead to its inactivation or silencing by methylation, causing accumulation of the transcription factor HIF (hypoxia inducible factor), hence upregulation of angiogenic and cell proliferation genes. Overexpressed proteins, such as PDGF and VEGF, are strong proangiogenic factors and are therapeutic targets that have led to the first reliable targeted treatments with bevacizumab and VEGF TKIs [111]. IMP-3 and VEGF-R2 correlate with poor prognosis in pRCC. IMP3, being expressed 10 times more in pRCC than in ccRCC, is particularly unfavorable and indicates a high risk of developing metastases [112].

Integrated data analysis of ccRCC evolution toward malignancy shows a shift in regulation of metabolic pathways controlled by genetic and epigenetic genes [113].

In RCCs, hypomethylation of DNA and miRNA-21 promoter, mutations in the chromosome $3 p 21$ locus of epigenetic genes, chromatin remodeling, BAP1, and SETD2 all cause 
inactivation of the TSG PBRM1 and predict an adverse outcome $[114,115]$.

In B-cell lymphoma, the serum levels of miRNA-155, miRNA-210, and miRNA-21 are significantly elevated [116]; miRNA-21 is also associated with prostate cancer [115] and represents a potential diagnostic biomarker in neoplasia [117]. In most cancers, alterations in chromosomal regions encoding for miRNAs which result in their altered expression have been reported, pointing to a causal role of miRNAs in cancer pathogenesis and metastasis [118]. To identify and validate circulating miRNAs for use as diagnostic and prognostic biomarkers, screening investigations were undertaken, revealing that high levels of miRNA-21 and miRNA-155 can be early indicators of lung cancer [119]. miRNA-205 and Lt$7 \mathrm{f}$ can be diagnostic-prognostic biomarkers of ovarian cancer [120].

Numerous genetic and epigenetic biomarkers that may accompany lung cancer development, such as miRNAs and autoantibodies against tumor-associated antigens (TAA), can be detected up to 5 years before NSCLC diagnosis [121]. Future studies should focus on the identification of miRNA signatures as biomarkers and on the development of targeted therapeutics based on miRNAs, as recently attempted for breast cancer [122]. Circulating regulatory noncoding RNAs, such as miRNAs, lncRNAs, and exosomes, are very promising [123].

Exosomes are nanodimensioned extracellular secreted vesicles, which are primarily involved in intracellular communications and transport of proteins, lipids, and other cell components, such as DNA and noncoding RNAs, functioning as signaling molecules, between cells. Free or exosomeencapsulated biomarkers are present in liquid biopsies and can be detected, even in traces, once concentrated and purified, or amplified from blood, urine, saliva, and other body fluids and exudates [124]. The latest generation of microarrays for gene expression profiling and miRNA transcriptomes has allowed a better assessment of changes in heterogeneity within and between tumors, for early prediction of therapeutic response, establishment of resistance, and metastatic evolution of the disease.

\section{Nanotechnology in Cancer Diagnostics}

Routine diagnostics lacks specificity and sensitivity. Engineering of nanoparticles (NPs) to specifically target cancer cells, combining diagnostic imaging with chemotherapeutic and/or physical anticancer agents, has facilitated the creation of multifunctional nanoplatforms for monitoring cancer therapy [125].

Novel nanodevices convert data from interactions between molecules and subcellular structures, based on mutations and alterations in pathways driving cells to malignant transformation, into transduced or amplified electromagnetic signals $[126,127]$. At the same time, they can be combined with more effective therapeutic treatments due to their reduced toxicity, as they only target the transformed cells [128].

This has been achieved, in the latest biosensors and nanovehicles (Table 2) [129], utilizing new nanomaterials and exploiting electric, magnetic, optical, acoustical, mechanical, chemical, and physical properties of unusual compounds in order to increase the sensitivity of diagnostic tools and the effectiveness of the therapeutic devices [130]. In addition, newly discovered genetic and epigenetic oncomarkers have been exploited [18] as molecular targets, either for early cancer detection [131] or for providing more effective and targeted therapies [132].

Modern nanooncology provides both more accurate tumor imaging and more efficient therapy by drug delivery or activation of other anticancer agents, selectively targeted to cancer cells on the basis of their genetic profile [126].

3.1. Gold, Silica, and Magnetic Nanoparticles. Gold nanoparticles (GNPs) are powerful imaging labels and contrast agents, due to their unusual optical properties, biocompatibility, absence of cytotoxicity, resistance to photobleaching, and absorbance at near-infrared light region (NIR) of surfaceenhanced Raman scattering (SERS) [133] and have been successfully used in both cancer diagnosis and therapy [127]. The absorption and scattering properties displayed by GNPs, conjugated to a $\mathrm{ScFv}$ antibody, have been used for in vivo targeting of EGFR in NSCLC cells, enabling spectroscopic detection of microscopic tumors.

Recently, plasmon resonance for in vivo molecular imaging of carcinogenesis [134] has been experimentally used in breast cancer cells, allowing precancerous epithelium detection and selective photothermal therapy of OSCC cells by anti-EGFR antibody-conjugated NPs [135]. Gold nanoparticles combined with gold nanorods conjugated with specific antibody facilitated extremely sensitive immunoassay detection of PSA in prostate cancer cells by dynamic light scattering (DLS) [136, 137].

Metal, magnetic, and silica nanoparticles (SiNPs) present unique thermophysical properties, such as encapsulated phase change, which make them particularly suitable for cancer-specific marker diagnosis [129]. This type of NPs behaves as a highly sensitive multiplex in oncomarker detection. Phase-change materials (PCM) are able to absorb thermal energy without increasing temperature during melting. Heat diffusion from the environment to the core of PCM is time-consuming and increases the melting peak at constant temperature. SiNPs take advantage of the thermic and physical features of silica, during phase changes. This allows restriction of any changes in metal composition inside the silica-encapsulated NPs, thus providing thermal barcodes with melting peaks directly related to the concentration of the target molecules. SiNPs labeled with fluorescence resonance energy transfer (FRET) dyes have been used for simultaneous and multiplexed detection in order to monitor the presence of cancer cells. In preclinical models of colon carcinoma, the efficient accumulation, in target tissues, of silica-polyethylene glycol nanoparticles (SPNs) functionalized with two metastasis-specific peptides results in specific imaging of tumor foci, providing a powerful fluorescent platform for image-detection of submillimetric colorectal micrometastases [138]. Aptamer-conjugated silica NPs specifically target T-cell leukemia and B-cell lymphoma. 


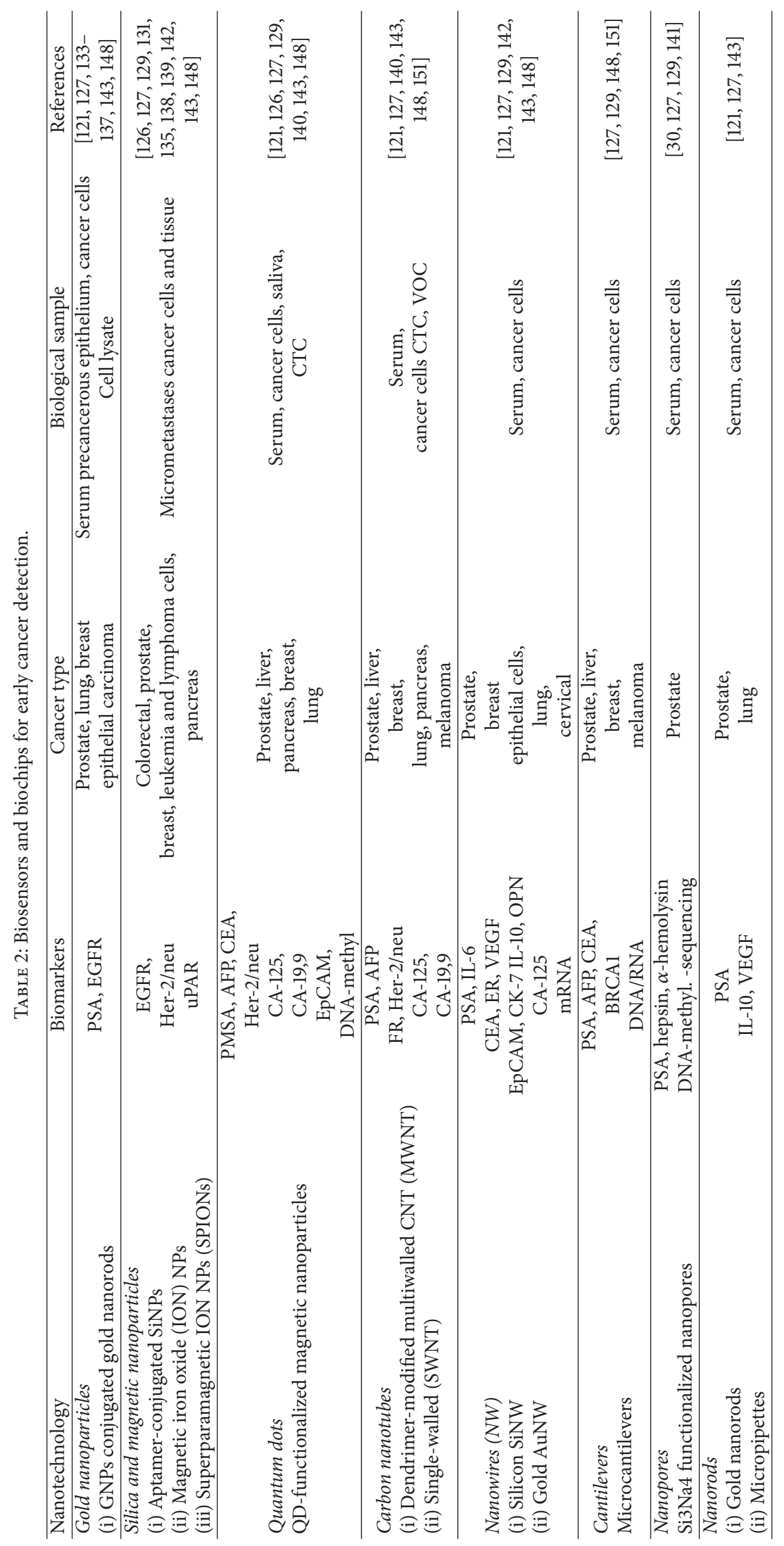


Magnetic NPs are particularly recommended in cancer cell imaging to develop diagnostic chips and in therapy to specifically release drugs within selectively targeted malignant cells [139]. Magnetic NPs are able to aggregate in the presence of target molecules, facilitating cancer biomarker measurement. Following surgery, locally implanted magnetic NPs can be left in loco; hence, this innovative method for detecting in vivo cancer biomarkers, such as EGFR and HER2/neu, may be also suitable for continuous monitoring. The ability of magnetic particles to aggregate to target cells via affinity ligands has been exploited as a chip-based NMR system and, with miniaturization and multiplexing, for simultaneously identifying various biomarkers in small liquid samples.

Magnetic nanosensors are three orders of magnitude more sensitive than current clinical assays and recent microchips have been developed that can reveal up to 64 different proteins at the same time.

3.2. Quantum Dots and Carbon Nanotubes. Quantum dots (QD) are semiconductors composed of light-emitting nanocrystals displaying a wide-band adsorption with emission bands scattered from UV to NIR [140]. QD-conjugated multifunctional NPs have been successfully used for combining DOX (doxorubicin) with fluorescent PSMA imaging for targeting prostate cancer cells [127]. Ultrasensitive nanomicrochips, based on QD-labeled antibodies for other specific biomarkers, such as CEA, CA-125, and HER2/neu, have been recently developed, enabling oncomarker detection in serum and saliva. QD-functionalized magnetic NPs have also facilitated cancer targeting and imaging. In this way, it has been possible to identify and discard CTC from the blood by magnetic separation, using immobilized biotinylated EpCAM antibodies targeting malignant cells. In addition, the ultrasensitive MS-qFRET nanotechnological assay has been developed to detect and quantify DNA methylation in blood samples. As DNA methylation is directly involved in carcinogenesis through silencing of TSGs, this method can predict the effectiveness of a specific cancer treatment.

Carbon nanotubes (CNT) are helical nanotubes of graphic carbon particularly recommended for label-free detection of several oncomarkers and, once conveniently modified, allow a series of extremely specific and sensitive diagnostic assays, for example, a multifunctional dendrimer-modified multiwalled CNT for targeting the folic acid (FA) receptor, which is overexpressed in cancer cells, a single-walled CNT coupled with electrochemiluminescent silica NPs for detecting PSA in the blood, and, finally, a multilayered enzyme-coated CNT for high sensitive chemiluminescent immunoassay of serum AFP. CNT, due to their intrinsic ability to penetrate the cell membrane in order to release drugs within targeted transformed cells and to convert optical energy into thermal energy, can be exposed to NIR in order to thermally destroy cancer cells. Conjugation of QD to CNT has enabled localizing cancer cells in the patients, by QD imaging, and subsequent cell destruction via drug release or thermal inactivation [140]. This approach can open up new horizons on multimodal nanoplatforms in oncology.
3.3. Nanowires and Cantilevers. In $2 \mathrm{O} 3$ nanowires (NW), silicon nanowires (SiNW), and gold-conducting polymer NW (AuNW) can be individually assembled or modified for detecting cancer biomarkers [127, 129], in particular, SiNWs for VEGF detection; peptide nucleic acid- (PNA-) modified SiNW for RNA cancer biomarkers; SiO2-NW IL-10 for alkaline phosphatase sandwich immune-assay of interleukin10; and osteopontin (OPN) lung cancer biomarkers. AuNW polymers were used as templates for CK-7, epithelial cell marker, enzymatic immunoassay, and polypyrrole- (Ppy-) NW, which were integrated into the field effect transistor (FET) system, as a semiconducting channel for the cancer antigen 125 (CA 125) assay.

Cantilevers are biosensors for detecting cancer proteins and DNA/RNA biomarkers, based on nanometer-scale produced bends, according to changes in their environment or surface. A cantilever microassay for PSA detection consists of laser measurement of bend deflection caused by antibodyantigen specific binding. In this assay, anti-PSA antibodyconjugation to specific linkers at the microcantilever surface permits reduction of background signals [129] and allows measurement of very low antigen concentrations, monitoring the surface stress by the reflected laser beam. The latest microassays for sensing cancer biomarkers have been developed exploiting resonant microcantilevers, allowing measurements of shifts in frequency that originate from antibody-antigen interactions. As decrease in shift depends on molecular interactions and is proportional to the target concentration, microcantilevers coated with piezoelectric film have been developed, which allow exact determination of CEA concentrations. Another powerful immunological method using integrated rotating resonance microcantilevers for detecting AFP in undiluted serum at nanogram $/ \mathrm{mL}$ level has allowed the investigation of liver cancer at a very early stage. Nanomechanical analysis for detecting cancer cells in vivo has been reported, which measures tumor cell-stiffness with respect to normal cells, by atomic force microscopy.

3.4. Nanopores and Nanorods. Nanopores have great potential for detecting biomolecules such as DNA, antigens, ions, and drugs [30]. Nanopores may consist of synthetic or artificial materials or may be protein-based. They are based on the principle that ionic conductance changes when micropores submitted to an electric field are passed through by small molecules; thus, the electrochemical impedance, which varies according to the concentration and the nature of the molecules, can be measured, allowing for the stochastic sensing and biophysical studies of individual molecules. Nanopore membrane functionalized with antibodies enables PSA and hepsin detection while the $\alpha$-hemolysin protein nanopore enables detecting immobilized DNA. Another possible application of nanopores is the detection of aberrant DNA methylation by epigenetic analysis [141]. Protein or synthetic nanopores in Si3N4 membranes have been used for methylated DNA detection and for ultrarapid DNA sequencing.

Finally, other ultrasensitive sensors for cancer biomarkers are the gold nanorods and micropipettes. Gold nanorods can be used as molecular imaging agent and, in conjugation 
with anti-PSA antibodies, can facilitate DLS analysis for the PSA immunoassay. Micropipettes, due to the capability of capturing antibodies on their surface, can be exploited for detecting IL-10 and VEGF [127].

\subsection{Multifunctional Nanoplatforms for Cancer Theranostics.} Over the past twenty years, a prodigious effort has been made in designing and developing nanometer-sized targeted probes for cancer diagnosis and therapy, which combine several features and diverse functions: stability in the circulation, accumulation to specific districts, responsiveness to local stimuli, and effective intracellular drug delivery and multimodality of action $[127,130]$. Magnetic iron oxide (ION) nanoparticles used as an MRI contrast agent in cancer imaging provide morphological details of the tumor and monitor in real-time the therapeutic response. Highly lymphotropic superparamagnetic iron oxide nanoparticles (SPIONs), which at once combine high-resolution MRI with optical imaging, have been successfully used for micrometastasis screening in patients with prostate cancer that have undergone surgical lymph node resection or biopsy [135].

Recently, multifunctional ultrasmall SPIONs (USPIONs) based on appropriate and biodegradable coating of N-phosphonomethyl iminodiacetic acid (PMIDA) and using functional hydrophilic, biocompatible, and spacer molecules, such as rhodamine B isothiocyanate, have been designed to combine cancer-targeted MRI/optical imaging and release of $\mathrm{pH}$-sensitive drugs [142]. By combining the aminederivatized with USPION-PMIDA support, very effective multimodal and theranostics nanoagents have been produced, which, when modified with both folic acid and methotrexate (MTX), simultaneously provide tumor imaging and intracellular drug targeting and delivery in tumors overexpressing folic acid receptors (FR) [128].

\section{Conclusion}

Diagnosis of cancer is often achieved at a late stage when the adverse effects and drawbacks limit conventional cancer therapy [126]. Novel molecular biomarkers offer new hope [143]; for example, miRNAs allow very early, precise, and innovative cancer diagnosis and therapy and improve prognosis $[144,145]$. Customized anticancer treatments started with specific antitumor mAbs and TKIs $[5,6]$; however, mAbs are frequently ineffective as second-line therapy [146], while TKIs easily engender resistance. To be effective, personalized oncology requires an anticancer approach at molecular level specifically directed to a single cancer cell. Hence, comprehensive diagnostics of the molecular signature of the tumor to be treated is essential. Orthotopic tumor models of patientderived xenograft (PDX) in immune-compromised mice allow predicting drug responsiveness and personalization of the anticancer treatment [147].

Current nanooncology is focused on satisfying the abovementioned diagnostic and therapeutic requirements. It is based on functionalized nanosensors and biochips for early and ultrasensitive detection of novel cancer biomarkers [127, 148] and/or on the latest NPs directed to specific cancer molecular targets, multifunctionalized for diagnostics imaging and targeting therapy $[125,128,132]$.

More complex systems have been developed to enhance drug delivery to key molecular structures in cancer cells and/ or neighboring tissues but also are able to operate as direct agents themselves, such as for thermal inactivation [149].

Second-generation polymeric nanoparticles have been approved or are undergoing evaluation. Modification of NPs facilitates the binding to a large number of cell ligands and optimizes physicochemical characteristics for drug targeting, release, and clearance [150]. The field of cancer theranostics is now dawning. Recently developed nanoparticles, functionalized to provide multifunctional nanoplatforms in melanoma [151], are potentially utilizable for many other cancer types [152, 153], including cancers that present a stem-cell phenotype [154]. Theranostics, being molecularly directed, can enable tumor detection at the level of a single cell(s) and can be simultaneously combined with tumor diagnostic imaging for monitoring very effective targeted therapy [155]. Dual diagnostic and therapeutic approach joined to specific targeting permits a real-time detection of nanovectors localized at the target-sites and direct visual appreciation of therapeutic effect on tumor cells, providing a feedback to diagnosis. To date, it was carried out only in animal models and cells, displaying a significant reduction and also the complete disappearance of the tumor mass [153]. Undoubtedly in the near future theranostics could provide the powerful tool for eradicating cancer and metastasis of any type, at every stage, offering the necessary and alternative follow-up supporting liquid biopsy, which represents a great future challenge for early tumor diagnostic.

Liquid biopsies have many clinical applications: the detection of circulating cell-free DNA (cfDNA) [124], CTC [64, 103], miRNAs, and exosomes [123]. Although with different modality and variable benefits it permits a very early and predictive cancer detection, in a noninvasive manner, through the characterization of the genetic profile of tumors that, especially under the selective pressure of treatment, often change their mutation pattern or acquire new mutations.

Very early stage cancer detection helps to reduce mortality, but this is conceivable only through widespread screening, since most of cancers are at the beginning asymptomatic.

Personalized oncology meets some of major expectations in diagnostics and therapy. However, genetic heterogeneity, inside and between tumors and metastasis, together with the instauration of resistance to antitumor agents represents the major challenge in targeting therapy of cancer and will require additional efforts.

At present, there is a lack of standard biomarkers for cancer diagnosis. Identification biomarkers at a very early stage of tumorigenesis could be further improved and other biomarkers specific for particular cancer type require confirmation. The development of biosensors for detecting serum biomarkers has provided high sensitive and specific noninvasive tool, making them extremely promising for use in early cancer diagnostics. Introduction of nanomaterials will adjust sensitivity of biosensors in microfluids rendering them more appropriate for high-throughput assay. In the 
near future, last generation biosensors will help to decrease mortality rate.

\section{Abbreviations}

ABL:

Abelson murine leukemic viral oncogene homolog

ADRP: $\quad$ Adipophilin

AFP: $\quad$ Alpha-fetoprotein

ALK: $\quad$ Anaplastic lymphoma receptor tyrosine

ALL: $\quad$ Acute lymphatic leukemia

AML: $\quad$ Acute myeloid leukemia

AMACR: Alpha-methylacyl-CoA racemase

APC: $\quad$ Adenomatous polyposis coli

A AQP1: $\quad$ Aquaporin 1

BCR:

BMP:

BRAF:

BAP1:

CA-125:

CDKNZA:

CEA:

cfDNA:

CIN:

CK:

CLL:

CML:

CIMP:

CRC:

CSCC:

CSC:

CTC:

CTLA-4:

DLS:

DNMT:

ECM:

EGFR:

EML4:

EMT:

FA:

FR:

FET:

FGFR:

FISH:

FOBT:

FRET:

GNPs:

GSTP1:

HCC:

HDAC:

Breakpoint cluster

Bone morphogenetic protein homolog B1

BRCA1 associated protein

Cancer antigen 125

Cyclin-dependent kinase

Carcinoembryonic antigen

Circulating cell-free DNA

Chromosomal instability

Cytokeratin

Chronic lymphocytic leukemia

Chronic myeloid leukemia

CpG dinucleotide island methylator phenotype

Colorectal cancer

Cervical squamous cell carcinoma

Cancer stem cell

Circulating tumor cells

Cytotoxic T-lymphocyte antigen-4

Dynamic light scattering

DNA methyltransferase

Extracellular matrix

Epidermal growth factor receptor

Echinoderm microtubule-associated

protein-like 4

Epithelial mesenchymal transition

Folic acid

Folic acid receptors

Field effect transistor

Fibroblast growth factor receptor

Fluorescent in situ hybridization

Fecal occult blood test

Fluorescence resonance energy transfer

Gold nanoparticles

Glutathione-S-transferase $\mathrm{p} 1$

HER2/ERBB2: Human epidermal growth factor receptor Human hepatocellular carcinoma

Histone deacetylase

\section{ERBB2}

HGFR/MET: Hepatocyte growth factor receptor c-MET

HIF:

Hypoxia inducible factor

$5 \mathrm{HmC:}$
HPV: Human papilloma virus

KIT: $\quad$ v-Kit Hardy-Zuckerman 4 feline sarcoma viral oncogene homolog

KRAS: Kirsten rat sarcoma viral oncogene homolog

LMO1: LIM domain only 1

IL-2: Interleukin-2

LCP1: L-plastin, lymphocyte cytosolic protein 1

lncRNAs: Long noncoding RNAs

mAbs: Monoclonal antibodies

MAPK: Mitogen-activated protein kinase

MDR: Multidrug resistance

MDS: $\quad$ Myelodysplastic syndromes

5mC: 5-Methylcytosine

MGMT: O6-Methylguanine-methyltransferase

miRNAs: MicroRNAs

MLH1: mutl homolog 1

MSC: Mesenchymal stem cells

MSI: Microsatellite instability

mTOR: Mammalian target of rapamycin

MTX: Methotrexate

NDN: Necdin gene

$\mathrm{NF} \kappa \mathrm{B}: \quad$ Nuclear factor kappa B

NIR: Near-infrared light region

NM23A: Nonmetastatic cells 1 protein

NMR: Nuclear magnetic resonance

NNMT: Nicotinamide, $\mathrm{N}$-methyltransferase

NMR: Nuclear magnetic resonance

NPs: Nanoparticles

NSCLC: Non-small-cell lung cancer

OR: Overall response

OSCC: Oral squamous cell carcinoma

PCM: Phase-change materials

PCNA: Proliferating cell nuclear antigen

PD-1: $\quad$ Programmed death-1

PDGF-R: Platelet derived growth factor receptor

PFS: $\quad$ Progression-free survival

PI3K: Phosphoinositide-3-kinase

PMSA: Prostate membrane specific antigen

PSA: Prostate specific antigen

PTEN: Phosphatase and tensin homolog

RA: Retinoid acid

RASSF: RAS-associated domain family

SCC-Ag: Squamous cell carcinoma antigen

SCLC: Small-cell lung cancer

SCNAs: Somatic copy-number alterations

SEPT9: Septin 9

SERS: $\quad$ Surface-enhanced Raman scattering

SNP: $\quad$ Single-nucleotide polymorphism

SPIONs: Superparamagnetic iron oxide nanoparticles

SiPNs: Silica nanoparticles

SPOP: Speckle-type POZ protein

TEPI2: $\quad$ Tissue-factor pathway inhibitor 2

TGF- $\beta$ : Tumor growth factor beta

TK: $\quad$ Tyrosine kinase

TKIs: Tyrosine kinase inhibitors

TFs: $\quad$ Transcription factors

TSC1: Tuberous sclerosis complex 1 gene 
TSG: Tumor suppressor gene

USPIONs: Ultrasmall SPIONs

VEGF-R: Vascular growth factor receptor

VHL: $\quad$ Von Hippel-Lindau

VOC: Volatile organic compounds

WNT: Wingless-related type.

\section{Competing Interests}

The authors declare that they have no competing interests.

\section{Acknowledgments}

Antonio Ponzetto is a recipient of an unrestricted grant from Rotfil SpA. The authors are indebted to Dr. Monica Rinaldi and Professor Bruno Azzarone for critically reading the paper. They would like to thank Dr. Radhika Srinivasan for her invaluable help with editing.

\section{References}

[1] D. G. de Castro, P. A. Clarke, B. Al-Lazikani, and P. Workman, "Personalized cancer medicine: molecular diagnostics, predictive biomarkers, and drug resistance," Clinical Pharmacology and Therapeutics, vol. 93, no. 3, pp. 252-259, 2013.

[2] H. L. McLeod, "Cancer pharmacogenomics: early promise, but concerted effort needed," Science, vol. 340, no. 6127, pp. 15631566, 2013.

[3] T. J. R. Harris and F. McCormick, "The molecular pathology of cancer," Nature Reviews Clinical Oncology, vol. 7, no. 5, pp. 251265, 2010.

[4] R. Pirker, F. J. F. Herth, K. M. Kerr et al., "Consensus for EGFR mutation testing in non-small cell lung cancer: results from a European workshop," Journal of Thoracic Oncology, vol. 5, no. 10, pp. 1706-1713, 2010.

[5] B. W. Newsome and M. S. Ernstoff, "The clinical pharmacology of therapeutic monoclonal antibodies in the treatment of malignancy; have the magic bullets arrived?" British Journal of Clinical Pharmacology, vol. 66, no. 1, pp. 6-19, 2008.

[6] N. Normanno, S. Tejpar, F. Morgillo, A. De Luca, E. Van Cutsem, and F. Ciardiello, "Implications for KRAS status and EGFRtargeted therapies in metastatic CRC," Nature Reviews Clinical Oncology, vol. 6, no. 9, pp. 519-527, 2009.

[7] R. R. Raval, A. B. Sharabi, A. J. Walker, C. G. Drake, and P. Sharma, "Tumor immunology and cancer immunotherapy: summary of the 2013 SITC primer," Journal for Immuno Therapy of Cancer, vol. 2, article 14, 2014.

[8] E. J. Lipson and C. G. Drake, "Ipilimumab: an anti-CTLA-4 antibody for metastatic melanoma," Clinical Cancer Research, vol. 17, no. 22, pp. 6958-6962, 2011.

[9] J. D. Wolchok, H. Kluger, M. K. Callahan et al., "Nivolumab plus Ipilimumab in advanced melanoma," The New England Journal of Medicine, vol. 369, no. 2, pp. 122-133, 2013.

[10] E. M. Van Allen, N. Wagle, A. Sucker et al., "The genetic landscape of clinical resistance to RAF inhibition in metastatic melanoma," Cancer Discovery, vol. 4, no. 1, pp. 94-109, 2014.

[11] T. G. Bivona, H. Hieronymus, J. Parker et al., "FAS and NF$\kappa \mathrm{B}$ signalling modulate dependence of lung cancers on mutant EGFR," Nature, vol. 471, no. 7339, pp. 523-526, 2011.
[12] P. B. Chapman, A. Hauschild, C. Robert et al., "Improved survival with vemurafenib in melanoma with BRAF V600E mutation," The New England Journal of Medicine, vol. 364, no. 26, pp. 2507-2516, 2011.

[13] A. Hauschild, J.-J. Grob, L. V. Demidov et al., "Dabrafenib in BRAF-mutated metastatic melanoma: a multicentre, openlabel, phase 3 randomised controlled trial," The Lancet, vol. 380, no. 9839, pp. 358-365, 2012.

[14] N. Wagle, E. M. Van Allen, D. J. Treacy et al., "MAP kinase pathway alterations in BRAF-mutant melanoma patients with acquired resistance to combined RAF/MEK inhibition," Cancer Discovery, vol. 4, no. 1, pp. 61-68, 2013.

[15] J. Villanueva, J. R. Infante, C. Krepler et al., "Concurrent MEK2 mutation and BRAF amplification confer resistance to BRAF and MEK inhibitors in melanoma," Cell Reports, vol. 4, no. 6, pp. 1090-1099, 2013.

[16] A. Marchetti, M. Del Grammastro, G. Filice et al., "Complex mutations \& subpopulations of deletions at exon 19 of EGFR in NSCLC revealed by next generation sequencing: potential clinical implications," PLoS ONE, vol. 7, no. 7, Article ID e42164, 2012.

[17] A. Marchetti, A. Ardizzoni, M. Papotti et al., "Recommendations for the analysis of ALK gene rearrangements in nonsmall-cell lung cancer: a consensus of the italian association of medical oncology and the Italian society of pathology and cytopathology," Journal of Thoracic Oncology, vol. 8, no. 3, pp. 352-358, 2013.

[18] H. Shen and P. W. Laird, "Interplay between the cancer genome and epigenome," Cell, vol. 153, no. 1, pp. 38-55, 2013.

[19] L. G. Biesecker and N. B. Spinner, "A genomic view of mosaicism and human disease," Nature Reviews Genetics, vol. 14, no. 5, pp. 307-320, 2013.

[20] J. G. Paez, P. A. Jänne, J. C. Lee et al., "EGFR mutations in lung cancer: correlation with clinical response to gefitinib therapy," Science, vol. 304, no. 5676, pp. 1497-1500, 2004.

[21] J. Malm, T. E. Fehniger, P. Danmyr et al., "Developments in biobanking workflow standardization providing sample integrity and stability," Journal of Proteomics, vol. 95, pp. 38-45, 2013.

[22] G. da Cunha Santos, N. Liu, M.-S. Tsao, S. Kamel-Reid, K. Chin, and W. R. Geddie, "Detection of EGFR and KRAS mutations in fine-needle aspirates stored on whatman FTA cards: is this the tool for biobanking cytological samples in the molecular era?" Cancer Cytopathology, vol. 118, no. 6, pp. 450-456, 2010.

[23] S. Subramaniam, R. K. Thakur, V. K. Yadav, R. Nanda, S. Chowdhury, and A. Agrawal, "Lung cancer biomarkers: state of the art," Journal of Carcinogenesis, vol. 12, no. 3, 2013.

[24] M. G. Kris, R. B. Natale, R. S. Herbst et al., "Efficacy of gefitinib, an inhibitor of the epidermal growth factor receptor tyrosine kinase, in symptomatic patients with non-small cell lung cancer: a randomized trial," The Journal of the American Medical Association, vol. 290, no. 16, pp. 2149-2158, 2003.

[25] A. Chi, S. Remick, and W. Tse, "EGFR inhibition in non-small cell lung cancer: current evidence and future directions," Biomarker Research, vol. 1, article 2, 2013.

[26] G. R. Oxnard, A. Binder, and P. A. Jänne, "New targetable oncogenes in non-small-cell lung cancer," Journal of Clinical Oncology, vol. 31, no. 8, pp. 1097-1104, 2013.

[27] S. Liu, L.-C. Wu, J. Pang et al., "Sp1/NF $\kappa$ B/HDAC/miR-29b regulatory network in KIT-driven myeloid leukemia," Cancer Cell, vol. 17, no. 4, pp. 333-347, 2010. 
[28] G. Iyer, A. J. Hanrahan, M. I. Milowsky et al., "Genome sequencing identifies a basis for everolimus sensitivity," Science, vol. 338, no. 6104 , p. 221, 2012.

[29] J. Shendure and H. Ji, "Next-generation DNA sequencing," Nature Biotechnology, vol. 26, no. 10, pp. 1135-1145, 2008.

[30] B. M. Venkatesan and R. Bashir, "Nanopore sensors for nucleic acid analysis," Nature Nanotechnology, vol. 6, no. 10, pp. 615-624, 2011.

[31] S. Valastyan and R. A. Weinberg, "Tumor metastasis: molecular insights and evolving paradigms," Cell, vol. 147, no. 2, pp. 275292, 2011.

[32] L. Wan, K. Pantel, and Y. Kang, "Tumor metastasis: moving new biological insights into the clinic," Nature Medicine, vol. 19, no. 11, pp. 1450-1464, 2013.

[33] D. F. Quail and J. A. Joyce, "Microenvironmental regulation of tumor progression and metastasis," Nature Medicine, vol. 19, no. 11, pp. 1423-1437, 2013.

[34] L.-M. Postovit, N. V. Margaryan, E. A. Seftor et al., "Human embryonic stem cell microenvironment suppresses the tumorigenic phenotype of aggressive cancer cells," Proceedings of the National Academy of Sciences of the United States of America, vol. 105, no. 11, pp. 4329-4334, 2008.

[35] F. G. Giancotti, "Mechanisms governing metastatic dormancy and reactivation," Cell, vol. 155, no. 4, pp. 750-764, 2013.

[36] J. Chou, J. H. Lin, A. Brenot, J.-W. Kim, S. Provot, and Z. Werb, "GATA3 suppresses metastasis and modulates the tumour microenvironment by regulating microRNA-29b expression," Nature Cell Biology, vol. 15, no. 2, pp. 201-213, 2013.

[37] Y. Zhang, P. Yang, T. Sun et al., "miR-126 and miR-126* repress recruitment of mesenchymal stem cells and inflammatory monocytes to inhibit breast cancer metastasis," Nature Cell Biology, vol. 15, no. 3, pp. 284-294, 2013.

[38] J. P. Thiery, "Epithelial-mesenchymal transitions in tumour progression," Nature Reviews Cancer, vol. 2, no. 6, pp. 442-454, 2002.

[39] D. M. Gonzalez and D. Medici, "Signaling mechanisms of the epithelial-mesenchymal transition," Science Signaling, vol. 7, no. 344, p. re8, 2014.

[40] L. A. Byers, L. Diao, J. Wang et al., "An epithelial-mesenchymal transition gene signature predicts resistance to EGFR and PI3K inhibitors and identifies Axl as a therapeutic target for overcoming EGFR inhibitor resistance," Clinical Cancer Research, vol. 19, no. 1, pp. 279-290, 2013.

[41] Z. Zhang, J. C. Lee, L. Lin et al., "Activation of the AXL kinase causes resistance to EGFR-targeted therapy in lung cancer," Nature Genetics, vol. 44, no. 8, pp. 852-860, 2012.

[42] D. C. Radisky and M. A. LaBarge, "Epithelial-mesenchymal transition and the stem cell phenotype," Cell Stem Cell, vol. 2, no. 6, pp. 511-512, 2008.

[43] R. K. Ambasta, A. Sharma, and P. Kumar, "Nanoparticle mediated targeting of VEGFR and cancer stem cells for cancer therapy," Vascular Cell, vol. 3, article 26, 2011.

[44] N. D. Marjanovic, R. A. Weinberg, and C. L. Chaffer, "Cell plasticity and heterogeneity in cancer," Clinical Chemistry, vol. 59, no. 1, pp. 168-179, 2013.

[45] S. J. Vidal, V. Rodriguez-Bravo, M. Galsky, C. Cordon-Cardo, and J. Domingo-Domenech, "Targeting cancer stem cells to suppress acquired chemotherapy resistance," Oncogene, vol. 33, no. 36, pp. 4451-4463, 2014.

[46] C. E. Eyler and J. N. Rich, "Survival of the fittest: cancer stem cells in therapeutic resistance and angiogenesis," Journal of Clinical Oncology, vol. 26, no. 17, pp. 2839-2845, 2008.
[47] H. Lou and M. Dean, "Targeted therapy for cancer stem cells: the patched pathway and ABC transporters," Oncogene, vol. 26, no. 9, pp. 1357-1360, 2007.

[48] R. Morrison, S. M. Schleicher, Y. Sun et al., "Targeting the mechanisms of resistance to chemotherapy and radiotherapy with the cancer stem cell hypothesis," Journal of Oncology, vol. 2011, Article ID 941876, 13 pages, 2011.

[49] S. Vinogradov and X. Wei, "Cancer stem cells and drug resistance: the potential of nanomedicine," Nanomedicine, vol. 7, no. 4, pp. 597-615, 2012.

[50] B. T. Kawasaki, E. M. Hurt, T. Mistree, and W. L. Farrar, "Targeting cancer stem cells with phytochemicals," Molecular Interventions, vol. 8, no. 4, pp. 174-184, 2008.

[51] P. B. Gupta, T. T. Onder, G. Jiang et al., "Identification of selective inhibitors of cancer stem cells by high-throughput screening," Cell, vol. 138, no. 4, pp. 645-659, 2009.

[52] Y. Zhao, D. Y. Alakhova, and A. V. Kabanov, "Can nanomedicines kill cancer stem cells?" Advanced Drug Delivery Reviews, vol. 65, no. 13-14, pp. 1763-1783, 2013.

[53] S. B. Baylin and P. A. Jones, "A decade of exploring the cancer epigenome-biological and translational implications," Nature Reviews Cancer, vol. 11, no. 10, pp. 726-734, 2011.

[54] J. Sana, P. Faltejskova, M. Svoboda, and O. Slaby, "Novel classes of non-coding RNAs and cancer," Journal of Translational Medicine, vol. 10, article 103, 2012.

[55] A. Hui, C. How, E. Ito, and F.-F. Liu, "Micro-RNAs as diagnostic or prognostic markers in human epithelial malignancies," $B M C$ Cancer, vol. 11, article 500, 2011.

[56] S. Fukushige and A. Horii, "DNA methylation in cancer: a gene silencing mechanism and the clinical potential of its biomarkers," Tohoku Journal of Experimental Medicine, vol. 229, no. 3, pp. 173-185, 2013.

[57] P. A. Jones, "Functions of DNA methylation: islands, start sites, gene bodies and beyond," Nature Reviews Genetics, vol. 13, no. 7, pp. 484-492, 2012.

[58] A. G. Knudson Jr., "Mutation and cancer: statistical study of retinoblastoma," Proceedings of the National Academy of Sciences of the United States of America, vol. 68, no. 4, pp. 820-823, 1971.

[59] G. A. Calin and C. M. Croce, "MicroRNA signatures in human cancers," Nature Reviews Cancer, vol. 6, no. 11, pp. 857-866, 2006.

[60] S. Liu, "Epigenetics advancing personalized nanomedicine in cancer therapy," Advanced Drug Delivery Reviews, vol. 64, no. 13, pp. 1532-1543, 2012.

[61] T. F. Gajewski, "Molecular profiling of melanoma and the evolution of patient-specific therapy," Seminars in Oncology, vol. 38, no. 2, pp. 236-242, 2011.

[62] K. L. Nathanson, "Using genetics and genomics strategies to personalize therapy for cancer: focus on melanoma," Biochemical Pharmacology, vol. 80, no. 5, pp. 755-761, 2010.

[63] P. V. Jithesh, J. M. Risk, A. G. Schache et al., "The epigenetic landscape of oral squamous cell carcinoma," British Journal of Cancer, vol. 108, no. 2, pp. 370-379, 2013.

[64] J. R. Schoenborn, P. Nelson, and M. Fang, "Genomic profiling defines subtypes of prostate cancer with the potential for therapeutic stratification," Clinical Cancer Research, vol. 19, no. 15, pp. 4058-4066, 2013.

[65] D. S. Uppal and S. M. Powell, "Genetics/genomics/proteomics of gastric adenocarcinoma," Gastroenterology Clinics of North America, vol. 42, no. 2, pp. 241-260, 2013. 
[66] C. P. Lange and P. W. Laird, "Clinical applications of DNA methylation biomarkers in colorectal cancer," Epigenomics, vol. 5, no. 2, pp. 105-108, 2013.

[67] H. Kim, K. Kim, S. J. Yu et al., "Development of biomarkers for screening hepatocellular carcinoma using global data mining and multiple reaction monitoring," PLoS ONE, vol. 8, no. 5, Article ID e63468, 2013.

[68] M. Chmelarová, E. Dvoráková, J. Špacek, J. Laco, M. MŽik, and V. Palicka, "Promoter methylation of GATA4, WIF1, NTRK1 and other selected tumour suppressor genes in ovarian cancer," Folia Biologica, vol. 59, no. 2, pp. 87-92, 2013.

[69] A. I. Ojesina, L. Lichtenstein, S. S. Freeman et al., "Landscape of genomic alterations in cervical carcinomas," Nature, vol. 506, no. 7488, pp. 371-375, 2013.

[70] A. Q. van Hoesel, Y. Sato, D. A. Elashoff et al., "Assessment of DNA methylation status in early stages of breast cancer development," British Journal of Cancer, vol. 108, no. 10, pp. 2033-2038, 2013.

[71] A. I. Oliveira, C. Jerónimo, and R. Henrique, "Moving forward in bladder cancer detection and diagnosis: the role of epigenetic biomarkers," Expert Review of Molecular Diagnostics, vol. 12, no. 8, pp. 871-878, 2012.

[72] S. Markopoulou, G. Nikolaidis, and T. Liloglou, "DNA methylation biomarkers in biological fluids for early detection of respiratory tract cancer," Clinical Chemistry and Laboratory Medicine, vol. 50, no. 10, pp. 1723-1731, 2012.

[73] K. A. Heichman and J. D. Warren, "DNA methylation biomarkers and their utility for solid cancer diagnostics," Clinical Chemistry and Laboratory Medicine, vol. 50, no. 10, pp. 17071721, 2012.

[74] J. K. Samuelsson, S. Alonso, F. Yamamoto, and M. Perucho, "DNA fingerprinting techniques for the analysis of genetic and epigenetic alterations in colorectal cancer," Mutation Research/Fundamental and Molecular Mechanisms of Mutagenesis, vol. 693, no. 1-2, pp. 61-76, 2010.

[75] G. S. Inamdar, S. V. Madhunapantula, and G. P. Robertson, “Targeting the MAPK pathway in melanoma: why some approaches succeed and other fail," Biochemical Pharmacology, vol. 80, no. 5, pp. 624-637, 2010.

[76] J. A. Gasche and A. Goel, "Epigenetic mechanisms in oral carcinogenesis," Future Oncology, vol. 8, no. 11, pp. 1407-1425, 2012.

[77] D. Hanahan and R. A. Weinberg, "Hallmarks of cancer: the next generation," Cell, vol. 144, no. 5, pp. 646-674, 2011.

[78] J. B. Rawson and B. Bapat, "Epigenetic biomarkers in colorectal cancer diagnostics," Expert Review of Molecular Diagnostics, vol. 12, no. 5, pp. 499-509, 2012.

[79] M. Berg and K. Søreide, "Genetic and epigenetic traits as biomarkers in colorectal cancer," International Journal of Molecular Sciences, vol. 12, no. 12, pp. 9426-9439, 2011.

[80] M.-A. Song, M. Tiirikainen, S. Kwee, G. Okimoto, H. Yu, and L. L. Wong, "Elucidating the landscape of aberrant DNA methylation in hepatocellular carcinoma," PLoS ONE, vol. 8, no. 2, Article ID e55761, 2013.

[81] A. Fujimoto, Y. Totoki, T. Abe et al., "Whole-genome sequencing of liver cancers identifies etiological influences on mutation patterns and recurrent mutations in chromatin regulators," Nature Genetics, vol. 44, no. 7, pp. 760-764, 2012.

[82] C. Ozen, G. Yildiz, A. T. Dagcan et al., "Genetics and epigenetics of liver cancer," New Biotechnology, vol. 30, no. 4, pp. 381-384, 2013.
[83] G. Diaz, M. Melis, A. Tice et al., "Identification of microRNAs specifically expressed in hepatitis $C$ virus-associated hepatocellular carcinoma," International Journal of Cancer, vol. 133, no. 4, pp. 816-824, 2013.

[84] L. E. De Faveri, C. D. Hurst, F. M. Platt et al., "Putative tumour suppressor gene necdin is hypermethylated and mutated in human cancer," British Journal of Cancer, vol. 108, no. 6, pp. 1368-1377, 2013.

[85] Y. Gui, G. Guo, Y. Huang et al., "Frequent mutations of chromatin remodeling genes in transitional cell carcinoma of the bladder," Nature Genetics, vol. 43, no. 9, pp. 875-878, 2011.

[86] B. R. Druliner, J. A. Fincher, B. S. Sexton et al., "Chromatin patterns associated with lung adenocarcinoma progression," Cell Cycle, vol. 12, no. 10, pp. 1536-1543, 2013.

[87] P. K. Vogt, "Retroviral oncogenes: a historical primer," Nature Reviews Cancer, vol. 12, no. 9, pp. 639-648, 2012.

[88] T. Soussi, "Advances in carcinogenesis: a historical perspective from observational studies to tumor genome sequencing and TP53 mutation spectrum analysis," Biochimica et Biophysica Acta-Reviews on Cancer, vol. 1816, no. 2, pp. 199-208, 2011.

[89] A. J. Bass, M. S. Lawrence, L. E. Brace et al., "Genomic sequencing of colorectal adenocarcinomas identifies a recurrent VTI1ATCF7L2 fusion," Nature Genetics, vol. 43, no. 10, pp. 964-970, 2011.

[90] A. Kreso, C. A. O’Brien, P. van Galen et al., "Variable clonal repopulation dynamics influence chemotherapy response in colorectal cancer," Science, vol. 339, no. 6119, pp. 543-548, 2013.

[91] D. Singh, J. M. Chan, P. Zoppoli et al., “Transforming fusions of FGFR and TACC genes in human glioblastoma," Science, vol. 337, no. 6099, pp. 1231-1235, 2012.

[92] S. Seton-Rogers, "Glioblastoma: transforming fusions induce aneuploidy," Nature Reviews Cancer, vol. 12, no. 9, article 585, 2012.

[93] V. Frattini, V. Trifonov, J. M. Chan et al., "The integrated landscape of driver genomic alterations in glioblastoma," Nature Genetics, vol. 45, no. 10, pp. 1141-1149, 2013.

[94] P. A. Northcott, D. J. Shih, J. Peacock et al., "Subgroup-specific structural variation across 1,000 medulloblastoma genomes," Nature, vol. 488, no. 7409, pp. 49-56, 2012.

[95] G. P. Tonini, A. Nakagawara, and F. Berthold, "Towards a turning point of neuroblastoma therapy," Cancer Letters, vol. 326, no. 2, pp. 128-134, 2012.

[96] M. Capasso, M. Devoto, C. Hou et al., "Common variations in BARD1 influence susceptibility to high-risk neuroblastoma," Nature Genetics, vol. 41, no. 6, pp. 718-723, 2009.

[97] K. Wang, S. J. Diskin, H. Zhang et al., "Integrative genomics identifies LMO1 as a neuroblastoma oncogene," Nature, vol. 469, no. 7329, pp. 216-220, 2011.

[98] M. Sausen, R. J. Leary, S. Jones et al., "Integrated genomic analyses identify ARID1A and ARID1B alterations in the childhood cancer neuroblastoma," Nature Genetics, vol. 45, no. 1, pp. 12-17, 2013.

[99] B. Banelli, D. F. Merlo, G. Allemanni, A. Forlani, and M. Romani, "Clinical potentials of methylator phenotype in stage 4 high-risk neuroblastoma: an open challenge," PLOS ONE, vol. 8, no. 5, Article ID e63253, 2013.

[100] L. Van Neste, J. G. Herman, G. Otto, J. W. Bigley, J. I. Epstein, and W. Van Criekinge, "The epigenetic promise for prostate cancer diagnosis," Prostate, vol. 72, no. 11, pp. 1248-1261, 2012.

[101] H.-Y. Yoon, Y.-W. Kim, H. W. Kang et al., "Pyrosequencing analysis of APC methylation level in human prostate tissues: 
a molecular marker for prostate cancer," Korean Journal of Urology, vol. 54, no. 3, pp. 194-198, 2013.

[102] M. Ilie, V. Hofman, E. Long et al., "Current challenges for detection of circulating tumor cells and cell-free circulating nucleic acids, and their characterization in non-small cell lung carcinoma patients. What is the best blood substrate for personalized medicine?" Annals of Translational Medicine, vol. 2, no. 11, p. 107, 2014.

[103] M. Ilie, V. Hofman, E. Long-Mira et al., “'Sentinel' circulating tumor cells allow early diagnosis of lung cancer in patients with Chronic obstructive pulmonary disease," PLoS ONE, vol. 9, no. 10, Article ID el11597, 2014.

[104] Y. J. Zhao, Q. Ju, and G. C. Li, “Tumor markers for hepatocellular carcinoma," Molecular and Clinical Oncology, vol. 1, no. 4, pp. 593-598, 2013.

[105] S.-Y. Liao, O. N. Aurelio, K. Jan, J. Zavada, and E. J. Stanbridge, "Identification of the MN/CA9 protein as a reliable diagnostic biomarker of clear cell carcinoma of the kidney," Cancer Research, vol. 57, no. 14, pp. 2827-2831, 1997.

[106] J. J. Morrissey, A. N. London, J. Luo, and E. D. Kharasch, "Urinary biomarkers for the early diagnosis of kidney cancer," Mayo Clinic Proceedings, vol. 85, no. 5, pp. 413-421, 2010.

[107] D. Su Kim, Y. D. Choi, M. Moon et al., "Composite three-marker assay for early detection of kidney cancer," Cancer Epidemiology Biomarkers and Prevention, vol. 22, no. 3, pp. 390-398, 2013.

[108] H. Abe and T. Kamai, "Recent advances in the treatment of metastatic renal cell carcinoma," International Journal of Urology, vol. 20, no. 10, pp. 944-955, 2013.

[109] S. G. C. Kroeze, A. M. Bijenhof, J. L. H. R. Bosch, and J. J. M. Jans, "Diagnostic and prognostic tissuemarkers in clear cell and papillary renal cell carcinoma," Cancer Biomarkers, vol. 7, no. 6, pp. 261-268, 2010.

[110] L. J. Costa and H. A. Drabkin, "Renal cell carcinoma: new developments in molecular biology and potential for targeted therapies," Oncologist, vol. 12, no. 12, pp. 1404-1415, 2007.

[111] M. Wehland, J. Bauer, N. E. Magnusson, M. Infanger, and D. Grimm, "Biomarkers for anti-angiogenic therapy in cancer," International Journal of Molecular Sciences, vol. 14, no. 5, pp. 9338-9364, 2013.

[112] W. G. Kaelin Jr., "The von Hippel-Lindau tumor suppressor protein and clear cell renal carcinoma," Clinical Cancer Research, vol. 13, no. 2, part 2, pp. 680s-684s, 2007.

[113] Cancer Genome Atlas Research Network, "Comprehensive molecular characterization of clear cell renal cell carcinoma," Nature, vol. 499, no. 7456, pp. 43-49, 2013.

[114] A. A. Hakimi, I. Ostrovnaya, B. Reva et al., "Adverse outcomes in clear cell renal cell carcinoma with mutations of 3 p21 epigenetic regulators BAP1 and SETD2: a report by MSKCC and the KIRC TCGA research network," Clinical Cancer Research, vol. 19, no. 12, pp. 3259-3267, 2013.

[115] P. S. Mitchell, R. K. Parkin, E. M. Kroh et al., "Circulating microRNAs as stable blood-based markers for cancer detection," Proceedings of the National Academy of Sciences of the United States of America, vol. 105, no. 30, pp. 10513-10518, 2008.

[116] C. H. Lawrie, S. Gal, H. M. Dunlop et al., "Detection of elevated levels of tumour-associated microRNAs in serum of patients with diffuse large B-cell lymphoma," British Journal of Haematology, vol. 141, no. 5, pp. 672-675, 2008.

[117] H. Qu, W. Xu, Y. Huang, and S. Yang, "Circulating miRNAs: promising biomarkers of human cancer," Asian Pacific Journal of Cancer Prevention, vol. 12, no. 5, pp. 1117-1125, 2011.
[118] S. Valastyan and R. A. Weinberg, "MicroRNAs: crucial multitasking components in the complex circuitry of tumor metastasis," Cell Cycle, vol. 8, no. 21, pp. 3506-3512, 2009.

[119] D. Zheng, S. Haddadin, Y. Wang et al., "Plasma microRNAs as novel biomarkers for early detection of lung cancer," International Journal of Clinical and Experimental Pathology, vol. 4, no. 6, pp. 575-586, 2011.

[120] H. Zheng, L. Zhang, Y. Zhao et al., "Plasma miRNAs as diagnostic and prognostic biomarkers for ovarian cancer," PLoS ONE, vol. 8, no. 11, Article ID e77853, 2013.

[121] Y. Zhang, D. Yang, L. Weng, and L. Wang, "Early lung cancer diagnosis by biosensors," International Journal of Molecular Sciences, vol. 14, no. 8, pp. 15479-15509, 2013.

[122] M. A. Cortez, J. W. Welsh, and G. A. Calin, "Circulating MicroRNAs as noninvasive biomarkers in breast cancer," Recent Results in Cancer Research, vol. 195, pp. 151-161, 2012.

[123] H. Schwarzenbach, N. Nishida, G. A. Calin, and K. Pantel, "Clinical relevance of circulating cell-free microRNAs in cancer," Nature Reviews Clinical Oncology, vol. 11, no. 3, pp. 145-156, 2014.

[124] E. Heitzer, P. Ulz, and J. B. Geigl, "Circulating tumor DNA as a liquid biopsy for cancer," Clinical Chemistry, vol. 61, no. 1, pp. 112-123, 2015.

[125] D. Peer, J. M. Karp, S. Hong, O. C. Farokhzad, R. Margalit, and R. Langer, "Nanocarriers as an emerging platform for cancer therapy," Nature Nanotechnology, vol. 2, no. 12, pp. 751-760, 2007.

[126] D. J. Bharali and S. A. Mousa, "Emerging nanomedicines for early cancer detection and improved treatment: current perspective and future promise," Pharmacology and Therapeutics, vol. 128, no. 2, pp. 324-335, 2010.

[127] Y.-E. Choi, J.-W. Kwak, and J. W. Park, "Nanotechnology for early cancer detection," Sensors, vol. 10, no. 1, pp. 428-455, 2010.

[128] R. Ranganathan, S. Madanmohan, A. Kesavan et al., "Nanomedicine: towards development of patient-friendly drug-delivery systems for oncological applications," International Journal of Nanomedicine, vol. 7, pp. 1043-1060, 2012.

[129] Y. Hu, D. H. Fine, E. Tasciotti, A. Bouamrani, and M. Ferrari, "Nanodevices in diagnostics," Wiley Interdisciplinary Reviews: Nanomedicine and Nanobiotechnology, vol. 3, no. 1, pp. 11-32, 2011.

[130] E.-K. Lim, E. Jang, K. Lee, S. Haam, and Y.-M. Huh, "Delivery of cancer therapeutics using nanotechnology," Pharmaceutics, vol. 5, no. 2, pp. 294-317, 2013.

[131] I. E. Tothill, "Biosensors for cancer markers diagnosis," Seminars in Cell and Developmental Biology, vol. 20, no. 1, pp. 55-62, 2009.

[132] K. K. Jain, "Advances in the field of nanooncology," $B M C$ Medicine, vol. 8, article 83, 2010.

[133] X. Qian, X.-H. Peng, D. O. Ansari et al., "In vivo tumor targeting and spectroscopic detection with surface-enhanced Raman nanoparticle tags," Nature Biotechnology, vol. 26, no. 1, pp. 8390, 2008.

[134] J. Aaron, N. Nitin, K. Travis et al., "Plasmon resonance coupling of metal nanoparticles for molecular imaging of carcinogenesis in vivo," Journal of Biomedical Optics, vol. 12, no. 3, Article ID 034007, 2007.

[135] I. H. El-Sayed, X. Huang, and M. A. El-Sayed, "Selective laser photo-thermal therapy of epithelial carcinoma using anti-EGFR antibody conjugated gold nanoparticles," Cancer Letters, vol. 239, no. 1, pp. 129-135, 2006. 
[136] X. Liu, Q. Dai, L. Austin et al., “A one-step homogeneous immunoassay for cancer biomarker detection using gold nanoparticle probes coupled with dynamic light scattering," Journal of the American Chemical Society, vol. 130, no. 9, pp. 2780-2782, 2008.

[137] V. Mani, B. V. Chikkaveeraiah, V. Patel, J. S. Gutkind, and J. F. Rusling, "Ultrasensitive immunosensor for cancer biomarker proteins using gold nanoparticle film electrodes and multienzyme-particle amplification," ACS Nano, vol. 3, no. 3, pp. 585-594, 2009.

[138] M. Soster, R. Juris, S. Bonacchi et al., “Targeted dual-color silica nanoparticles provide univocal identification of micrometastases in preclinical models of colorectal cancer," International Journal of Nanomedicine, vol. 7, pp. 4797-4807, 2012.

[139] H. Lee, E. Sun, D. Ham, and R. Weissleder, "Chip-NMR biosensor for detection and molecular analysis of cells," Nature Medicine, vol. 14, no. 8, pp. 869-874, 2008.

[140] S. Y. Madani, F. Shabani, M. V. Dwek, and A. M. Seifalian, "Conjugation of quantum dots on carbon nanotubes for medical diagnosis and treatment," International Journal of Nanomedicine, vol. 8, pp. 941-950, 2013.

[141] J. Clarke, H.-C. Wu, L. Jayasinghe, A. Patel, S. Reid, and H. Bayley, "Continuous base identification for single-molecule nanopore DNA sequencing," Nature Nanotechnology, vol. 4, no. 4, pp. 265-270, 2009.

[142] M. Das, D. Mishra, P. Dhak et al., "Biofunctionalized, phosphonate-grafted, ultrasmall iron oxide nanoparticles for combined targeted cancer therapy and multimodal imaging," Small, vol. 5, no. 24, pp. 2883-2893, 2009.

[143] S. K. Arya and S. Bhansali, "Lung cancer and its early detection using biomarker-based biosensors," Chemical Reviews, vol. 111, no. 11, pp. 6783-6809, 2011.

[144] J. C. Brase, D. Wuttig, R. Kuner, and H. Sültmann, "Serum microRNAs as non-invasive biomarkers for cancer," Molecular Cancer, vol. 9, p. 306, 2010.

[145] D. Barh, A. Carpi, M. Verma et al., Cancer Biomarkers: Minimal and Noninvasive Early Diagnosis and Prognosis, CRC Press, Boca Raton, Fla, USA, 2014.

[146] K. T. Adams, "Ups and downs at ASCO 2014," Managed Care, vol. 23, no. 8, pp. 50-52, 2014.

[147] D. Siolas and G. J. Hannon, "Patient-derived tumor xenografts: transforming clinical samples into mouse models," Cancer Research, vol. 73, no. 17, pp. 5315-5319, 2013.

[148] A. Gdowski, A. P. Ranjan, A. Mukerjee, and J. K. Vishwanatha, "Nanobiosensors: role in cancer detection and diagnosis," Advances in Experimental Medicine and Biology, vol. 807, pp. 3358, 2014.

[149] L. Saenz del Burgo, J. L. Pedraz, and G. Orive, "Advanced nanovehicles for cancer management," Drug Discovery Today, vol. 19, no. 10, pp. 1659-1670, 2014.

[150] V. Sanna, N. Pala, and M. Sechi, “Targeted therapy using nanotechnology: focus on cancer," International Journal of Nanomedicine, vol. 9, no. 1, pp. 467-483, 2014.

[151] J. Chen, R. Shao, X. D. Zhang et al., "Applications of nanotechnology for melanoma treatment, diagnosis, and theranostics," International Journal of Nanomedicine, vol. 8, pp. 2677-2688, 2013.

[152] C. Esposito, A. Crema, A. Ponzetto, G. Murtas, and G. Carloni, "Multifunctional anti-cancer nano-platforms are moving to clinical trials," Current Drug Metabolism, vol. 14, no. 5, pp. 583604, 2013
[153] M. S. Muthu, D. T. Leong, L. Mei, and S.-S. Feng, "Nanotheranostics-application and further development of nanomedicine strategies for advanced theranostics," Theranostics, vol. 4, no. 6, pp. 660-677, 2014.

[154] A. R. Burke, R. N. Singh, D. L. Carroll, F. M. Torti, and S. V. Torti, "Targeting cancer stem cells with nanoparticle-enabled therapies," Journal of Molecular Biomarkers \& Diagnosis, supplement 8, 2012.

[155] M. K. Yu, J. Park, and S. Jon, “Targeting strategies for multifunctional nanoparticles in cancer imaging and therapy," Theranostics, vol. 2, no. 1, pp. 3-44, 2012. 

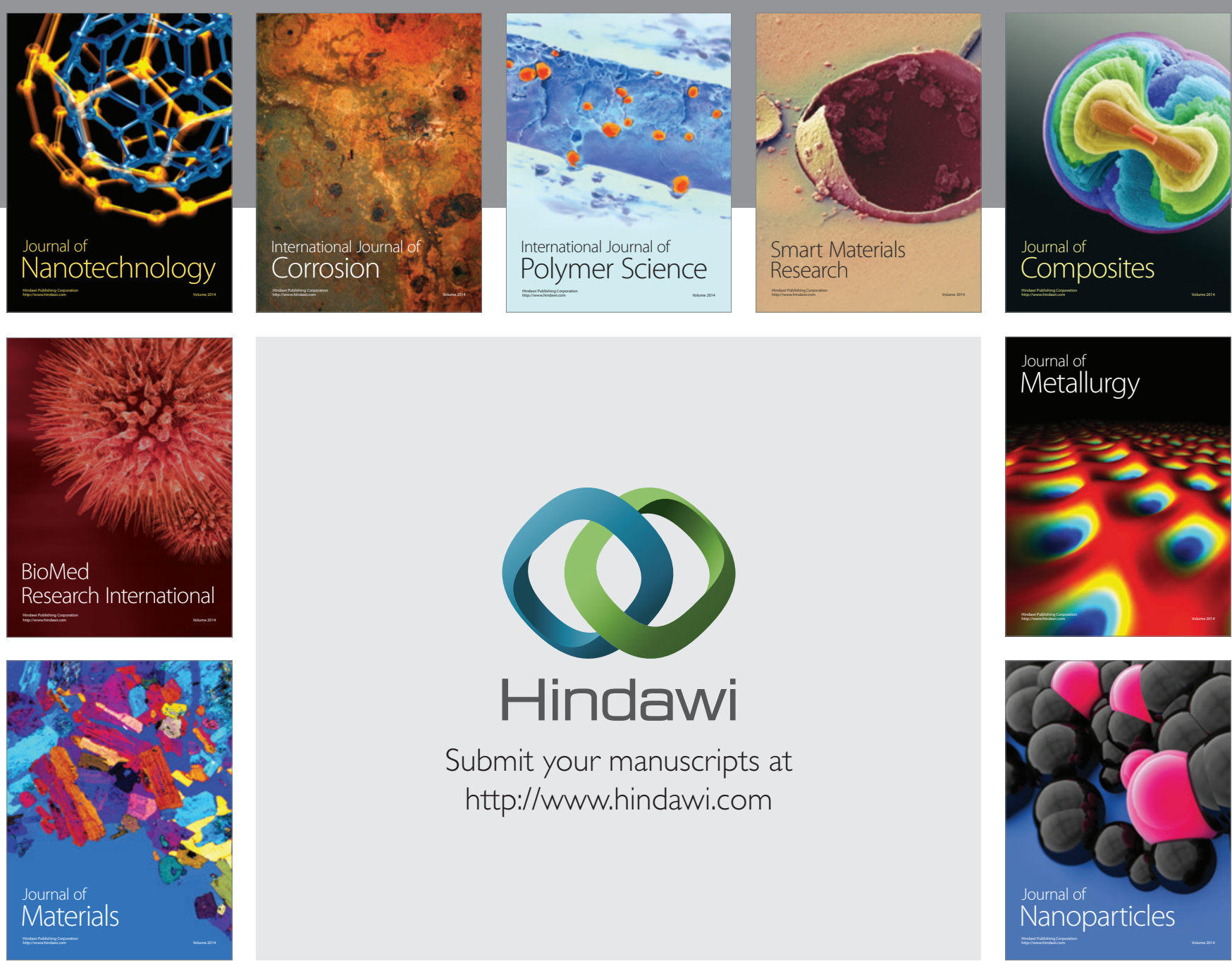

\section{Hindawi}

Submit your manuscripts at

http://www.hindawi.com

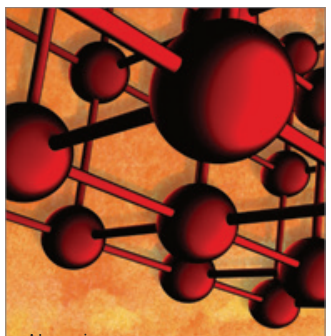

Materials Science and Engineering
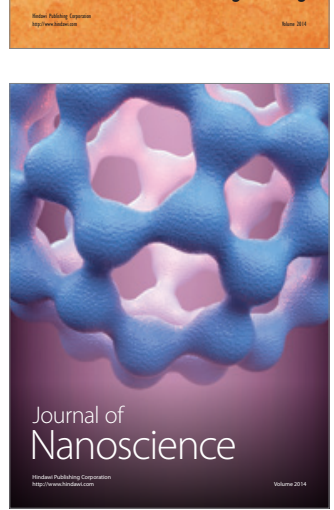
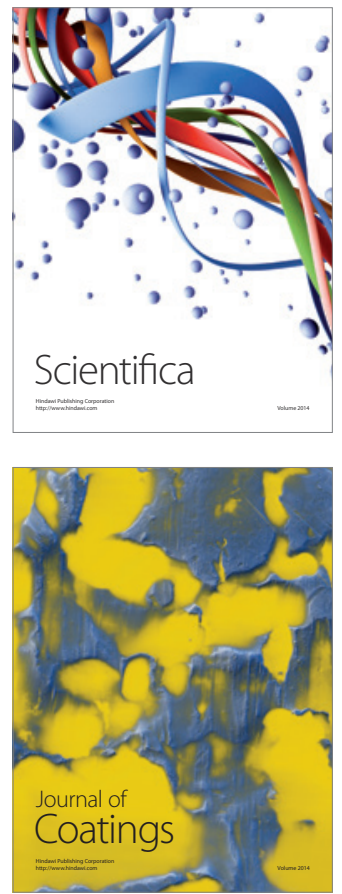
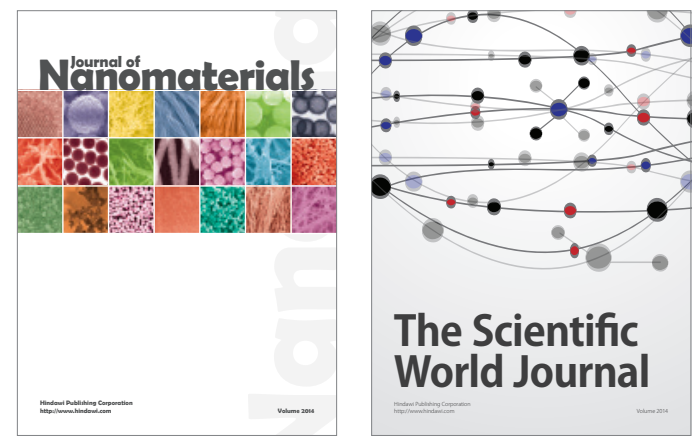

The Scientific World Journal
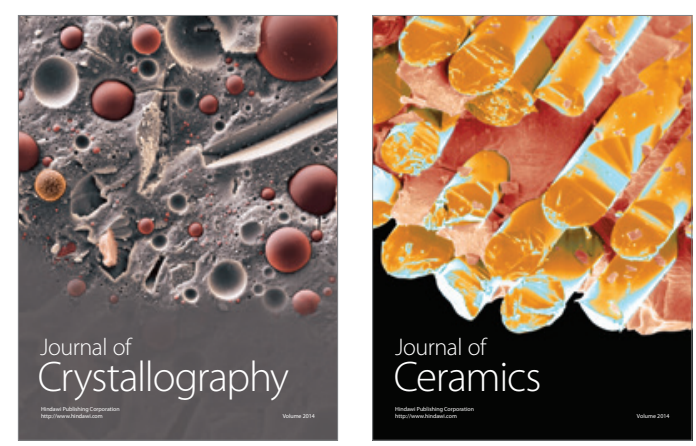
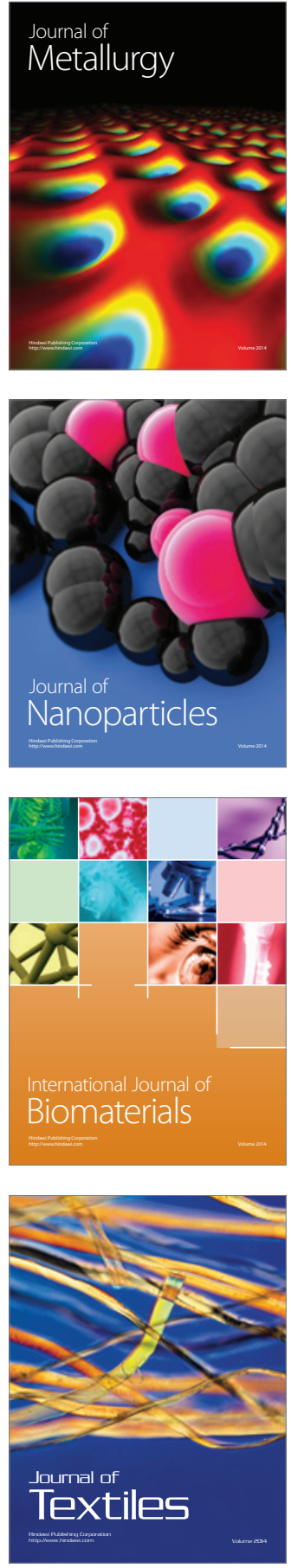